\title{
CONVERGENCE OF POLARIZATIONS, TORIC DEGENERATIONS, AND NEWTON-OKOUNKOV BODIES
}

\author{
MARK HAMILTON, MEGUMI HARADA, AND KIUMARS KAVEH
}

\begin{abstract}
Let $X$ be a smooth irreducible complex algebraic variety of dimension $n$ and $L$ a very ample line bundle on $X$. Given a toric degeneration of $(X, L)$ satisfying some natural technical hypotheses, we construct a deformation $\left\{J_{s}\right\}$ of the complex structure on $X$ and bases $\mathcal{B}_{s}$ of $H^{0}\left(X, L, J_{s}\right)$ so that $J_{0}$ is the standard complex structure and, in the limit as $s \rightarrow \infty$, the basis elements approach dirac-delta distributions centered at Bohr-Sommerfeld fibers of a moment map associated to $X$ and its toric degeneration. The theory of NewtonOkounkov bodies and its associated toric degenerations shows that the technical hypotheses mentioned above hold in some generality. Our results significantly generalize previous results in geometric quantization which prove "independence of polarization" between Kähler quantizations and real polarizations. As an example, in the case of general flag varieties $X=G / B$ and for certain choices of $\lambda$, our result geometrically constructs a continuous degeneration of the (dual) canonical basis of $V_{\lambda}^{*}$ to a collection of dirac delta functions supported at the Bohr-Sommerfeld fibres corresponding exactly to the lattice points of a Littelmann-Berenstein-Zelevinsky string polytope $\Delta_{\underline{w}_{0}}(\lambda) \cap \mathbb{Z}^{\operatorname{dim}(G / B)}$.
\end{abstract}

\section{CONTENTS}

1. Introduction

2. Statement of the main theorem 4

3. Variation of complex structures and bases of holomorphic sections

4. Proof of the main theorem

5. Toric degenerations coming from valuations and Newton-Okounkov bodies 24

References

\section{INTRODUCTION}

The motivation for the present manuscript arose from two rather different research areas: the theory of geometric quantization in symplectic geometry on the one hand, and the algebraic-geometric theory of Newton-Okounkov bodies - particularly in its relation to representation theory - on the other. Since we do not expect all readers of this paper to be familiar with both theories, we begin with a brief description of each.

We begin with a sketch of geometric quantization. As is well-known, symplectic geometry (Hamiltonian flows on symplectic manifolds) is the mathematical language for formulating classical physics, whereas it is

Date: March 2, 2018.

2010 Mathematics Subject Classification. Primary: 53D50, 14D06; Secondary: 14M25.

Key words and phrases. geometric quantization, polarization, integrable system, toric degeneration, degeneration of Kähler structure, valuations, Newton-Okounkov bodies.

The first author is partially supported by an NSERC Discovery Grant.

The second author is partially supported by an NSERC Discovery Grant and a Canada Research Chair (Tier 2) Award.

The third author is partially supported by a National Science Foundation Grant (Grant ID: DMS-1601303), Simons Foundation Collaboration Grant for Mathematicians, and Simons Fellowship award. 
the language of linear algebra and representation theory (unitary flows on Hilbert spaces) which forms the basis for formulating quantum physics. It has been a long-standing question within symplectic geometry to understand, from a purely mathematical and geometric perspective, the relation between the classical picture and the quantum picture, in terms of both the phase spaces and the defining equations of the dynamics. In one direction, to go from "quantum" to "classical", one can "take a classical limit". The reverse direction, i.e. that of systematically associating to a symplectic manifold $(M, \omega)$ a Hilbert space $Q(M, \omega)$ and to similarly relate, for instance, Hamilton's equations on $(M, \omega)$ to Schrödinger-type equations on $Q(M, \omega)$, is generally referred to as the theory of quantization. In this manuscript, we deal specifically with geometric quantization, a theory which associates to a symplectic manifold $(M, \omega)$ a Hilbert space $Q(M, \omega)$.

For a fixed $(M, \omega)$, it turns out that there are many possible ways of constructing a suitable Hilbert space $Q(M, \omega)$. To describe the choices we first set some notation. First suppose that $[\omega]$ is an integral cohomology class. Next, let $(L, \nabla, h)$ be a Hermitian line bundle with connection satisfying $\operatorname{curv}(\nabla)=\omega$. Such a triple is called a pre-quantum line bundle, or sometimes a pre-quantization. Also required is a polarization, of which the two main types are as follows. A Kähler polarization is a choice of compatible complex structure $J$ on $M$. Given such a $J$, one can define the quantization $Q(M, \omega)$ to be $H^{0}(M, L, J)$, the space of holomorphic sections of $L$ with respect to this complex structure $J$. On the other hand, one may also consider a (possibly singular) real polarization of $M$, which is a foliation of $M$ into Lagrangian submanifolds. Among the Lagrangian leaves one can define a special (usually finite, if $M$ is compact) subset called the Bohr-Sommerfeld leaves. There is not yet an agreed-upon "correct" definition of the corresponding Hilbert space for a real polarization, but one approach which has been investigated, and which will be used in this manuscript, is to consider distributional sections supported on the set of Bohr-Sommerfeld leaves. Based on the above discussion, the following natural question arises: Is the quantization $Q(M, \omega)$ "independent of polarization," i.e., independent of the choices made? More specifically, we can ask: does the quantization coming from a Kähler polarization agree with the quantization coming from a real polarization? The results of this manuscript confirms independence of polarization in a rather large class of examples, significantly extending previously known results which were restricted to special cases such as toric varieties and flag varieties.

We next briefly motivate the theory of Newton-Okounkov bodies. The famous Atiyah-Guillemin-Sternberg and Kirwan convexity theorems link equivariant symplectic and algebraic geometry to the combinatorics of polytopes. In the case of a toric variety $X$, the combinatorics of its moment map polytope $\Delta$ fully encodes the geometry of $X$, but this fails in the general case. In his influential work, Okounkov constructed (circa roughly 1996), for an (irreducible) projective variety $X \subseteq \mathbb{P}(V)$ equipped with an action of a reductive algebraic group $G$, a convex body $\tilde{\Delta}$ and a natural projection from $\tilde{\Delta}$ to the moment polytope $\Delta$ of $X$. Moreover, the volumes of the fibers of this projection encode the asymptotics of the multiplicities of the irreducible representations appearing in the homogeneous coordinate ring of $X$, or in other words, the Duistermaat-Heckman measure [18, 19]. Recently, Askold Khovanskii and the third author (also independently Lazarsfeld and Mustata [15]) vastly generalized Okounkov's ideas [11], and in particular constructed such $\tilde{\Delta}$ (called Newton-Okounkov bodies or sometimes simply Okounkov bodies) even without presence of any group action. In the setting studied by Okounkov, the maximum possible (real) dimension of the NewtonOkounkov body $\tilde{\Delta}$ is the transcendence degree of $\mathbb{C}(X)^{U}$ where $U$ is a maximal unipotent subgroup of $G$; when there is no group action (as in the setting studied in [11, 15]) we have $\operatorname{dim}_{\mathbb{R}}(\tilde{\Delta})=\operatorname{dim}_{\mathbb{C}}(X)$. Hence one interpretation of the results of Okounkov, Lazarsfeld-Mustata and Kaveh-Khovanskii is that there is a convex geometric/combinatorial object of 'maximal' dimension associated to $X$, even when $X$ is not a toric variety. This represents a vast expansion of the possible settings in which combinatorial methods may be used to analyze the geometry of algebraic varieties. There is promise of a rich theory which interacts with a wide range of inter-related areas: for instance, the third author showed [10] that the Littelmann-BerensteinZelevinsky string polytopes from representation theory, which generalize the well-known Gel'fand-Cetlin polytopes, are examples of $\tilde{\Delta}$. In the long-term, one can expect further applications to Schubert calculus and to geometric representation theory (e.g. see [13]). 
We now turn attention to the present manuscript. Firstly we should explain that the two seemingly disparate research areas mentioned above are related due to the results in [8], which uses a certain toric degeneration that arises from (the semigroup associated to) a Newton-Okounkov body [2] to construct integrable systems 11 on a wide class of projective varieties. Integrable systems are highly special Hamiltonian systems on symplectic (or, in our setting, Kähler) manifolds, and naturally give rise to (singular) real polarizations. Therefore, the theory of Newton-Okounkov bodies and their associated toric degenerations provide a natural setting in which to examine the theory of geometric quantization.

Before describing the statement of our main result (Theorem 2.3) in more detail we first recall the content of two manuscripts of Baier, Florentino, Mourao, and Nunes [3] and the first author and Konno [7], on which much of the current manuscript is based.

As already mentioned, a natural question that arises in the theory of geometric quantization is that of independence of polarization, i.e., the isomorphism class of a geometric quantization should be independent of the choices made. In the above context, this means we wish to show $\operatorname{dim} H^{0}(X, L, J)$ is equal to the number of Bohr-Sommerfeld fibres. Nunes and his collaborators initiated a "convergence of polarizations" approach to this question. Specifically, they deform the complex structure on $X$ in such a way that the Kähler polarization it defines converges, in a suitable sense to be further explained in Section 2 , to the real polarization on the same manifold. (See [17] for an overview of this program.) Although there are more general versions of this theory, in this paper we focus particularly on the case of symplectic toric manifolds as described in [3], where the Kähler polarization converges to the (singular) real polarization given by the fibres of the moment map (i.e., the integrable system) for a torus action. Indeed, for the case of a symplectic toric manifold $X$ associated to a Delzant polytope $\Delta$, it is well-known (see for example [6]) that there is a natural basis $\left\{\sigma^{m} \mid m \in \Delta \cap \mathbb{Z}^{n}\right\}$ of the space $H^{0}(X, L, J)$ of holomorphic sections of $L$ that is indexed by the integer lattice points in $\Delta$. It is also well-known that the Bohr-Sommerfeld fibres in $X$ are exactly the moment map fibres over precisely the same set of integer lattice points $\Delta \cap \mathbb{Z}^{n}$. In particular, the dimensions of the two quantizations agree. This is often seen as one of the most basic and motivational examples of the phenomenon of "independence of polarization".

The first author and Konno extend the results of Baier, Florentino, Mourao, and Nunes [3], which only apply to toric manifolds, to the case of the complete flag variety $\mathcal{F}$ lags $\left(\mathbb{C}^{n}\right)$ by making use of a toric degeneration of $\mathcal{F}$ lags $\left(\mathbb{C}^{n}\right)$ as constructed in [14]. The precise definition of a toric degeneration is given in Section 2; roughly, it is a (flat) family of algebraic varieties over $\mathbb{C}$ with generic fiber isomorphic to a given variety (in this case $\mathcal{F}$ lags $\left(\mathbb{C}^{n}\right)$ ) and special fiber a toric variety. The gradient-Hamiltonian-flow technique pioneered by Ruan [20] allows one to "pull back" the integrable system on the special fiber to one on the original variety $\mathcal{F}$ lags $\left(\mathbb{C}^{n}\right)$ and also enables the authors to apply the techniques of [3] to $\mathcal{F}$ lags $\left(\mathbb{C}^{n}\right)$.

With the above as motivation, we now describe the main result of this manuscript, although we do not give the full and precise statement due to its rather technical nature. Let $X$ be a smooth, irreducible complex algebraic variety with $\operatorname{dim}_{\mathbb{C}}(X)=n$, equipped with prequantum data $(L, \nabla, h)$. Suppose $X$ admits a toric degeneration $X$ as above (and made precise in Section 2). Under these assumptions, we can construct from the toric degeneration $X$ an integrable system $\mu: X \rightarrow \mathbb{R}^{n}$ on $X$ as in [8]. Very roughly, the main result of this paper then states the following (see Theorem 2.3 for the precise statement).

Theorem A. Under some natural technical hypotheses on $X$ and its toric degeneration $X$, there exists a continuous deformation $\left\{J_{s}\right\}_{s \in[0, \infty)}$ of the complex structure on (the underlying smooth manifold of) $X$ such that $J_{0}$ is the original complex structure on $X$, and in the limit as $s$ goes to $\infty$, the Kähler polarization defined by $J_{s}$ converges to the (singular) real polarization associated to the integrable system $\mu$ on $X$.

A notable feature of the above theorem is that, following the work of [3,7], Theorem A gives an explicit correspondence between specific elements of the Kähler and the real quantization (rather than just an equality of dimensions). The theorem above places additional hypotheses on $X$ and its toric degeneration,

\footnotetext{
${ }^{1}$ Here we use the term "integrable system" in the slightly non-standard sense of [8, Definition 1.1].
} 
so one immediately then asks: when do these hypotheses hold? The second result of this manuscript, made precise in Theorem [5.5, is that the construction given in [8] gives a large class of examples on which Theorem A applies, with the caveat that it is necessary to replace the original line bundle $L$ with a suitable tensor power (or, equiv galently, the original symplectic form with a positive integer multiple thereof). Roughly, our result (Theorem [5.5) states the following.

Theorem B. Let $X$ be as above, equipped with the line bundle $L$. Then the construction of the toric degeneration given by valuations (as in [8]) can be made to satisfy the additional technical hypotheses in Theorem A for the pair $\left(X, L^{\otimes d}\right)$ for sufficiently large $d$ and thus gives 'convergence of polarization' in these cases.

It is also worth mentioning that, in the precise statement of our main Theorem A, as given in Theorem 2.3 below, we assert an existence of a certain basis of holomorphic sections with appropriate convergence properties, which then implies Theorem A. In the general case considered in Theorem A, this basis is not very explicit for some of the values of the deformation parameter. However, in the situation of Theorem B where the toric degeneration arises from a valuation as above, we additionally show in Theorem [5.6 that this basis can be chosen to be both natural and explicit throughout the deformation.

As already mentioned, there are several indications of interesting connections between the theory of Newton-Okounkov bodies and representation theory. Indeed, putting the results of [10] and [8] together, we obtain an integrable system on a flag variety $G / B$ whose moment map image is precisely the LittelmannBerenstein-Zelevinsky string polytope $\Delta_{\underline{w}_{0}}(\lambda)$. This construction uses the so-called (dual) canonical basis of $V_{\lambda}^{*}$, the dual space of the $G$-module $V_{\lambda}$ with highest weight $\lambda$. The elements of this basis are parametrized by the lattice points $\Delta_{\underline{w}_{0}}(\lambda) \cap \mathbb{Z}^{\operatorname{dim}(G / B)}$. The integrable system gives rise to a real polarization of $L_{\lambda} \rightarrow G / B$ whose Bohr-Sommerfeld fibers are in one-to-one correspondence with $\Delta_{\underline{w}_{0}}(\lambda) \cap \mathbb{Z}^{\operatorname{dim}(G / B)}$, where $L_{\lambda}$ is the usual pullback line bundle from the Plücker embedding associated to weight $\lambda$. Moreover, in this context, the Borel-Weil theorem implies that the Kähler quantization $H^{0}\left(G / B, L_{\lambda}\right)$ can be identified with $V_{\lambda}^{*}$. Hence, in this special case and for sufficiently large multiples of $\lambda$, our Theorem A geometrically constructs a continuous degeneration of a basis of $V_{\lambda}^{*}$ to a collection of dirac delta functions supported at the Bohr-Sommerfeld fibres corresponding exactly to the lattice points $\Delta_{\underline{w}_{0}}(\lambda) \cap \mathbb{Z}^{\operatorname{dim}(G / B)}$.

The paper is organized as follows. In Section 2 we recall the necessary definitions and give a full and precise statement of our main Theorem 2.3. We set up the necessary family of complex structures, based largely on the work of [3] and [7], in Section 3] The proof of Theorem [2.3 occupies Section 4 We then show in Section 5 that the construction in [8] gives many examples of toric degenerations satisyfing the hypotheses of Theorem 2.3 .

We close with some brief comments on open questions. Firstly, we believe that the proof of our main results can be modified to work for an embedding of the toric degeneration into $Y \times \mathbb{C}$ where $Y$ is any smooth projective toric variety (instead of just a projective space). Secondly, we also believe that the constructions in this paper should descend to a GIT quotient by a torus action. We leave these open for future work.

Acknowledgements. We thank Yael Karshon for providing the opportunity for us to learn about each other's past work, thus helping to initiate this collaboration.

\section{STATEMENT OF THE MAIN THEOREM}

This section is devoted to the full and precise formulation of both the hypotheses for, and the statement of, our main theorem. We first provide a quick overview of geometric quantization and then dive straight into the technicalities of our theorem. Some key motivational remarks, which may aid a reader unfamiliar with this material, are contained in Remark 2.4.

We begin with definitions in the theory of geometric quantization. For details see e.g. [22]. Let $(X, \omega)$ be a symplectic manifold, i.e. $X$ is a smooth manifold and $\omega$ is a closed non-degenerate differential 2 -form on $X$. Suppose $(L, h, \nabla)$ is a complex line bundle with Hermitian structure $h$ and a connection $\nabla$ satisfying 
$\operatorname{curv}(\nabla)=\omega$ and with parallel transport preserving $h$. Such a triple is called a prequantum line bundle (or sometimes prequantum data, or prequantization) of $(X, \omega)$. Note that for a prequantum line bundle to exist, $[\omega]$ must be an integral cohomology class.

To pass from a prequantization to a quantization, we must choose a polarization, which is an integrable complex Lagrangian distribution on $X$. We only deal with two types of polarizations in this manuscript, as follows. Firstly, a Kähler polarization on $(X, \omega)$ is a compatible complex structure $J$. Then the corresponding Kähler quantization of $X$ is defined to be the space of holomorphic sections $H^{0}(X, L)$ of $L$, where $L$ is equipped with the holomorphic structure specified by $J$. Secondly, a (singular) real polarization on $X$ is a (singular) foliation of $X$ into Lagrangian submanifolds. Let $P$ denote the distribution in $T X$ corresponding to a real polarization. By abuse of language, we frequently refer to both the foliation and the distribution as a real polarization. A special case, of much recent interest in this area, is the (singular) foliation given by the fibres of a completely integrable system $F: X \rightarrow \mathbb{R}^{n}$. In this setting, a section $\sigma$ of $\left.L\right|_{U}$ over some open set $U \subset X$ is said to be flat along the leaves or leafwise flat if it is covariantly constant with respect to $\nabla$ in directions tangent to $P$. Leafwise flat sections always exist locally, but not usually globally. A leaf $\ell$ of the real polarization $P$ is a Bohr-Sommerfeld leaf if there exists a (nonzero) section $\sigma$ that is flat along the leaves and defined on all of $\ell$. The set of Bohr-Sommerfeld leaves is typically discrete in the space of leaves.

There is not at present a single agreed-upon definition of quantization using a real polarization. The basic philosophy is that the quantization corresponding to a real polarization "should" be given by leafwise flat sections over Bohr-Sommerfeld fibres, but since there are no globally defined leafwise flat sections (the set of Bohr-Sommerfeld fibers being usually discrete), this is not straightforward. One possible approach is to relax the requirement that the sections be smooth and look at distributional sections supported on the set of Bohr-Sommerfeld leaves. Several examples have been investigated using this approach; see [17] and references therein. This is also the approach we take in this manuscript.

We now set up the terminology and notation required for the statement of our main theorem. Let $X$ be a smooth, irreducible complex algebraic variety with $\operatorname{dim}_{\mathbb{C}}(X)=n$. We suppose in addition that $X$ is equipped with prequantum data $(\omega, J, L, h, \nabla)$ as above, where $(\omega, J)$ is a Kähler structure on $X$ and $L$ is a very ample line bundle over $X$ with Chern class equal to the Kähler class (i.e. $c_{1}(L)=[\omega] \in H^{2}(X, \mathbb{Z})$ ). In [8], using ideas from [16], a toric degeneration is used to construct an integrable system on $X$ which is a Hamiltonian $T^{n}$-action on an open dense subset of $X$. Recall that a toric degeneration of $X$ in the sense of [8] is a flat family $\pi: X \rightarrow \mathbb{C}$ of irreducible varieties such that the family is trivial over $\mathbb{C}^{*}=\mathbb{C} \backslash\{0\}$ with each fiber isomorphic to $X$, and the (possibly singular) central fiber $X_{0}:=\pi^{-1}(0)$ is a toric variety with respect to a complex torus $\mathbb{T}_{0}$. In particular there exists a fiber-preserving isomorphism $\varrho: X \times \mathbb{C}^{*} \rightarrow \pi^{-1}\left(\mathbb{C}^{*}\right)$ from the trivial fiber bundle $X \times \mathbb{C}^{*} \rightarrow \mathbb{C}^{*}$ to $\pi^{-1}\left(\mathbb{C}^{*}\right)$ and it follows by assumption on $X$ that $X$ is smooth away from $X_{0}$. For a fixed $t \in \mathbb{C}^{*}$, let $X_{t}:=\pi^{-1}(t)$ denote the fiber of the family $X$ and let $\varrho_{t}$ denote the restriction of $\varrho$ to $X \times\{t\}$. By assumption, $\varrho_{1}$ is an isomorphism from $X \cong X \times\{t\}$ to $X_{1}$. We will frequently identify $X$ with $X_{1}$ using this isomorphism $\varrho_{1}$.

In this manuscript, following [8] we assume that $X$ admits a toric degeneration with the additional property that the family $X$ can be embedded in $\mathcal{P} \times \mathbb{C}$ (where $\mathcal{P} \cong \mathbb{P}^{N}$ is a projective space for an appropriate choice of $N$ ), as an algebraic subvariety such that

(a) the map $\pi: X \rightarrow \mathbb{C}$ is the restriction to $X$ of the usual projection $\mathcal{P} \times \mathbb{C} \rightarrow \mathbb{C}$ to the second factor, and

(b) the action of $\mathbb{T}_{0}$ on $X_{0}$ extends to a linear action of $\mathbb{T}_{0}$ on $\mathcal{P} \cong \mathcal{P} \times\{0\}$.

Sometimes by abuse of notation we think of $X_{t} \subseteq \mathcal{P} \times\{t\}$ as a subvariety of $\mathcal{P}$ via the natural identification $\mathcal{P} \cong \mathcal{P} \times\{t\}$. Next we equip the ambient projective space $\mathcal{P}$ with prequantum data $\left(\omega_{\mathcal{P}}, J_{\mathcal{P}}, L_{\mathcal{P}} \cong\right.$ $\left.\mathcal{O}(1), \nabla_{\mathcal{P}}, h_{\mathcal{P}}\right)$. In addition to the prequantum data on $\mathcal{P}$, we need data on $\mathcal{P} \times \mathbb{C}$. We let $\Omega=\left(\omega_{\mathcal{P}}, \omega_{s t d}\right)$ denote the product Kähler structure on $\mathcal{P} \times \mathbb{C}$ where $\omega_{\text {std }}$ is the standard symplectic structure $\frac{i}{2} d z \wedge d \bar{z}$ on $\mathbb{C}$ with respect to the usual complex coordinate $z$. Moreover, by pulling back via the projection $\pi_{1}: \mathcal{P} \times \mathbb{C} \rightarrow \mathcal{P}$ to the first factor, we also have a line bundle $\pi_{1}^{*} L_{\mathcal{P}}$ on $\mathcal{P} \times \mathbb{C}$; this restricts to a line bundle $L_{x}$ on the family $X$. Let $\omega_{t}:=\left.\Omega\right|_{X_{t}}$ (respectively $L_{t}:=\left.L_{X}\right|_{X_{t}}$ ) denote the restriction of $\Omega$ (respectively $L_{X}$ ) to the fiber 
$X_{t}=\pi^{-1}(t)$. Moreover, pulling back the prequantum data on $\mathcal{P}$ to $\mathcal{P} \times \mathbb{C}$ via $\pi_{1}$ and restricting to $X$ yields prequantum data on $X$. Let $\nabla_{t}$ and $h_{t}$ denote the restrictions of $\nabla_{x}$ and $h x$, respectively, to the fiber $X_{t}$. With this notation in place we can state further assumptions on our toric degeneration (also see [8]):

(c) Under the isomorphism $\varrho_{1}: X \rightarrow X_{1}$, the prequantum data $\left(\omega_{1}, L_{1}, \nabla_{1}, h_{1}\right)$ on $X_{1}$ pulls back to the prequantum data $(\omega, L, \nabla, h)$ on $X$.

(d) The Kähler form $\Omega$ on $\mathcal{P} \times \mathbb{C}$ is $T_{0}$-invariant, where $T_{0} \cong\left(S^{1}\right)^{n}$ is the compact torus subgroup of the complex torus $\mathbb{T}_{0} \cong\left(\mathbb{C}^{*}\right)^{n}$ acting on the toric variety $X_{0}$.

In this context, it was shown in [8] that $X$ admits an integrable system which is a Hamiltonian torus action on an open dense subset of $X$. We quote the following.

Theorem 2.1. [8. Theorem (A) in Introduction] Let $X$ be a smooth, irreducible complex algebraic variety with $\operatorname{dim}_{\mathbb{C}}(X)=n$ equipped with a Kähler structure $\omega$. Let $\pi: X \rightarrow \mathbb{C}$ be a toric degeneration of $X$ in the sense described above. Suppose that $\pi: X \rightarrow \mathbb{C}$ additionally satisfies assumptions $(a)-(d)$. Then:

(1) there exists a surjective continuous map $\phi: X \rightarrow X_{0}$ which is a symplectomorphism on a dense open subset $U \subset X$ (in the classical topology),

(2) there exists a completely integrable system $\mu=\left(F_{1}, \ldots, F_{n}\right)$ on $(X, \omega)$ such that its moment map image $\Delta$ coincides with the moment map image of $\left(X_{0}, \omega_{0}\right)$ (which is a polytope).

(3) Let $U \subset X$ be the open dense subset of $X$ from (1). Then the integrable system $\mu=\left(F_{1}, \ldots, F_{n}\right)$ generates a Hamiltonian torus action on $U$, and the inverse image $\mu^{-1}\left(\Delta^{\circ}\right)$ of the interior of $\Delta$ under the moment map $\mu: X \rightarrow \mathbb{R}^{n}$ of the integrable system lies in the open subset $U$.

The main result of the present manuscript extends the above result by additionally working with the prequantum data. We first state one additional assumption on the family $X$. Since $\mathcal{P} \cong \mathbb{P}^{N}$ is a standard projective space, there is a complex torus $\mathbb{T}_{\mathcal{P}} \cong\left(\mathbb{C}^{*}\right)^{N}$ acting in the standard fashion on $\mathcal{P}$. By assumption (b) above, the torus $\mathbb{T}_{0}$ acting on $X_{0}$ extends to a linear action on $\mathcal{P}$, i.e. there is an inclusion homomorphism $\iota: \mathbb{T}_{0} \hookrightarrow \mathbb{T}_{\mathcal{P}}$ inducing the action of $\mathbb{T}_{0}$ on $\mathcal{P}$, and this action preserves $X_{0} \subset \mathcal{P}$. Similarly there is an inclusion (by abuse of notation also denoted $\iota$ ) of compact subgroups $\iota: T_{0} \cong\left(S^{1}\right)^{n} \hookrightarrow T_{\mathcal{P}} \cong\left(S^{1}\right)^{N}$. Let $\iota^{*}: \mathfrak{t}_{\mathcal{P}}^{*} \rightarrow \mathfrak{t}_{0}^{*}$ denote the corresponding dual projection. Let $\Delta_{\mathcal{P}} \subseteq \mathfrak{t}_{\mathcal{P}}^{*}$ denote the moment polytope (i.e. the moment map image) of $\mathcal{P}$ associated to the Hamiltonian action on $\mathcal{P}$ of $T_{\mathcal{P}}$, and let $\Delta_{0} \subseteq \mathfrak{t}_{0}^{*}$ denote the moment polytope of $X_{0}$ with respect to $\mathbb{T}_{0}$. We will make the following genericity assumption on $X_{0}$ :

(e) the special fiber $X_{0} \subseteq \mathcal{P}$ of our toric degeneration is the closure of the $\mathbb{T}_{0}$-orbit through [1:1:1: $\cdots: 1] \in \mathcal{P}$.

From this it follows by standard Hamiltonian-geometry arguments that $\iota^{*}\left(\Delta_{\mathcal{P}}\right)=\Delta_{0}$. We now define

$$
W_{0}:=\iota^{*}\left(\Delta_{\mathcal{P}} \cap \mathbb{Z}^{N}\right) \subseteq \Delta_{0} \cap \mathbb{Z}^{n} .
$$

Remark 2.2. It is not necessarily the case that $\iota^{*}\left(\Delta_{\mathcal{P}} \cap \mathbb{Z}^{N}\right)=\Delta_{0} \cap \mathbb{Z}^{n}$ even if $\iota^{*}\left(\Delta_{\mathcal{P}}\right)=\Delta_{0}$, as can be seen from the case when $X_{0}$ is the closure of the image of the embedding $\mathbb{C}^{*} \rightarrow \mathbb{P}^{2}$ given by $t \mapsto\left[t^{2}: t^{3}: 1\right]$.

We call an element of $W_{0}$ an interior point if it is in the interior if $\Delta_{0}$, and a boundary point if it is on the boundary of $\Delta_{0}$. We will show in Proposition 3.13 that $W_{0}$ is the Bohr-Sommerfeld set of the integrable system defined in Theorem 2.1

We can now state the main result of this paper.

Theorem 2.3. Let $X$ be a smooth irreducible complex algebraic variety with $\operatorname{dim}_{\mathbb{C}}(X)=n$. Suppose $X$ is equipped with prequantum data $(\omega, J, L, h, \nabla)$ as above. Let $\pi: X \rightarrow \mathbb{C}$ be a toric degeneration of $X$ satisfying assumptions (a)-(e). Let $\mu: X \rightarrow \mathbb{R}^{n}$ denote the integrable system associated to the toric degeneration $X$ as in Theorem [2.1. We additionally assume the following properties hold.

(f) The restriction map $H^{0}\left(\mathcal{P}, L_{\mathcal{P}}\right) \rightarrow H^{0}(X, L)$ (where we identify $\left.X \cong X_{1}, L \cong L_{1}\right)$ is surjective.

(g) The restriction to the respective lattices $\iota^{*}:\left(\mathfrak{t}_{\mathcal{P}}^{*}\right)_{\mathbb{Z}} \rightarrow\left(\mathfrak{t}_{0}^{*}\right)_{\mathbb{Z}}$ of the dual projection is surjective. 
(h) The dimension of the space of holomorphic sections of $L \rightarrow X$ is the cardinality of $W_{0}$, i.e. $\operatorname{dim}_{\mathbb{C}}\left(H^{0}(X, L)\right)=$ $\left|W_{0}\right|$.

Then there exists a continuous one-parameter family $\left\{J_{s}\right\}_{s \in[0, \infty)}$ of complex structures on the underlying $C^{\infty}$ manifold of $X$ such that the following holds.

- For $s=0$, the complex structure $J_{0}$ agrees with the original complex structure on $X$.

- For each $s \in[0, \infty)$ the triple $\left(X, \omega, J_{s}\right)$ is Kähler and the Hermitian line bundle $(L, h, \nabla)$ induces a holomorphic structure $\bar{\partial}^{s}$ on $L$.

- For each $s \in[0, \infty)$ there exists a basis $\left\{\sigma_{s}^{m} \mid m \in W_{0}\right\}$ of $H^{0}\left(X, L, \bar{\partial}^{s}\right)$ such that for all interior points $m \in W_{0}$ the section $\frac{\sigma_{s}^{m}}{\left\|\sigma_{s}^{m}\right\|_{L^{1}(X)}}$ converges to a delta function supported on the Bohr-Sommerfeld fiber $\mu^{-1}(m)$ in the following sense: there exist a covariantly constant section $\delta_{m}$ of $(L, X, \nabla)_{\mu^{-1}(m)}$ and a measure $d \theta_{m}$ on $\mu^{-1}(m)$ such that, for any smooth section $\tau$ of the dual line bundle $L^{*}$ over $X$, we have:

$$
\lim _{s \rightarrow \infty} \int_{X}\left\langle\tau, \frac{\sigma_{s}^{m}}{\left\|\sigma_{s}^{m}\right\|_{L^{1}(X)}}\right\rangle d(v o l)=\int_{\mu^{-1}(m)}\left\langle\tau, \delta_{m}\right\rangle d \theta_{m}
$$

where $\|\cdot\|_{L^{1}(X)}$ denotes the $L^{1}$-norm with respect to the symplectic volume.

Remark 2.4. The essential idea behind Theorem 2.3 is a construction due to Baier, Florentino, Mourao, and Nunes [3] of a varying set $\left\{\chi_{s}\right\}_{s \in[0, \infty)}$ (to be defined in Section (3) of diffeomorphisms of the underlying smooth manifold of the ambient projective space $\mathcal{P}$ which is designed to have certain convergence properties. Specifically, let $\left\{\sigma^{m}\right\}_{m \in \Delta_{\mathfrak{p}} \cap \mathbb{Z}^{n}}$ denote the natural basis of $H^{0}\left(\mathcal{P}, L_{\mathcal{P}}\right)$ already mentioned above (see e.g. [6]) with respect to the original complex structure. In [3] the authors construct the diffeomorphisms $\chi_{s}$ precisely so that a pullback $\sigma_{s}^{m}$ of $\sigma^{m}$, defined using the $\chi_{s}$ at time $s$, has the form (for large enough s) of a "bell curve" centred at the Bohr-Sommerfeld fiber $\mu_{\mathcal{P}}^{-1}(m)$ and, as $s \rightarrow \infty$ and with appropriate normalizations, the bell curve gets narrower and narrower, thus converging to a dirac-delta distribution supported on the Bohr-Sommerfeld fiber. The bulk of the arguments in the present paper are devoted to taking this fundamental construction of [3] for the projective space $\mathcal{P}$ and making the necessary adjustments to apply them to our more general situation. We rely heavily on [7], which already worked out some of the necessary steps for the case of the full flag variety.

Remark 2.5. - For $m \in W_{0}$ a boundary point, we are confident that similar arguments will show that the support of the section $\sigma_{s}^{m}$ localizes around the Bohr-Sommerfeld fiber $\mu^{-1}(m)$; doing so in this paper, however, would require including many more details of the constructions in [3] and [7] than we felt was desirable. On the other hand, for the full statement of the convergence of sections, we do not have a sufficiently concrete topological description of the fiber $\mu^{-1}(m)$ to construct an analogue of the measure $d \theta_{m}$ for fibres over boundary points of $W_{0}$.

- The normalization factor $\left\|\sigma_{s}^{m}\right\|_{L^{1}(X)}$ in (2.2) guarantees that the "area under the bell curve" mentioned in Remark 2.4 is always equal to 1 as $s$ varies.

- There are different versions of convergence in functional analysis, and the notion used in Theorem 2.3 is called "weak convergence". In particular, note that our convergence assertion is not uniform in the space of test sections $\tau$.

As mentioned in the introduction, the purpose of Sections 3 and 4 is to prove Theorem 2.3 We show that the theory of Newton-Okounkov bodies and their associated toric degenerations provides a large class of examples satisfying the hypotheses of Theorem 2.3 in Section 5.

\section{VARIATION OF COMPLEX STRUCTURES AND BASES OF HOLOMORPHIC SECTIONS}

In order to prove Theorem 2.3 we rely on work of Guillemin and Abreu [1, 4, 5] and, more recently, of Baier, Florentino, Mourao, and Nunes [3]. Moreover, the first author and Konno [7] have results similar to our Theorem 2.3 for the special case of flag manifolds and its Gel'fand-Tsetlin integrable system. In this 
section we recall the relevant background and establish the preliminary results required to prove the results in our (more general) case.

3.1. The gradient-Hamiltonian flow. Let $X$ be a smooth, irreducible complex algebraic variety and $\pi$ : $X \rightarrow \mathbb{C}$ be a toric degeneration of $X$ satisfying assumptions (a)-(d) as above. We equip (the smooth locus of) $X$ with the Kähler form $\omega_{x}:=\Omega \mid x$ as in Section 2 . The proof of our main result will use the gradientHamiltonian techniques of [8] which we now briefly recall.

Following Ruan [20], we define the gradient-Hamiltonian vector field corresponding to $\pi$ on the smooth locus $X_{\text {smooth }}$ of $X$ as follows. Let $\nabla(\operatorname{Re}(\pi))$ denote the gradient vector field on $X_{\text {smooth }}$ associated to the real part $\operatorname{Re}(\pi)$, with respect to the Kähler metric $\omega_{x}$. Since $\omega_{x}$ is Kähler and $\pi$ is holomorphic, the CauchyRiemann equations imply that $\nabla(\operatorname{Re}(\pi))$ is related to the Hamiltonian vector field $\xi_{\operatorname{Im}(\pi)}$ of the imaginary part $\operatorname{Im}(\pi)$ with respect to $\omega_{x}$ by

$$
\nabla(\operatorname{Re}(\pi))=-\xi_{\operatorname{Im}(\pi)} .
$$

Let $Z$ denote the closed subset of $X$ which is the union of the singular locus of $X$ and the critical set of $\operatorname{Re}(\pi)$, i.e. the set on which $\nabla(\operatorname{Re}(\pi))=0$. The gradient-Hamiltonian vector field $V_{\pi}$, which is defined only on the open set $X \backslash Z$, is by definition

$$
V_{\pi}:=-\frac{\nabla(\operatorname{Re}(\pi))}{\|\nabla(\operatorname{Re}(\pi))\|^{2}} .
$$

Where defined, $V_{\pi}$ is smooth. For $t \in \mathbb{R}_{\geq 0}$ let $\phi_{t}$ denote the time- $t$ flow corresponding to $V_{\pi}$. Note that since $V_{\pi}$ may not be complete, $\phi_{t}$ for a given $t$ is not necessarily defined on all of $X \backslash Z$; this issue is dealt with in the next proposition.

The gradient-Hamiltonian flow is the tool which allows us to relate the geometry of different fibres of the toric degeneration. Recall that $X_{t}$ denotes the fiber $\pi^{-1}(t)$ and that we often identify $X_{1}$ with the original variety $X$ using the isomorphism $\varrho_{1}$ above. We now record some facts, which hold under our assumptions, assembled from [8, Sections 2-4] and which are also used in the proof of [8, Theorem (A)].

Proposition 3.1. In the setting above, we have the following.

(a) Let $s, t \in \mathbb{R}$ with $s \geq t>0$. Where defined, the flow $\phi_{t}$ takes $X_{s} \cap(X \backslash Z)$ to $X_{s-t}$. In particular, where defined, $\phi_{t}$ takes a point $x \in X_{t}$ to a point in the fiber $X_{0}$. Moreover, for $s>t>0$, the map $\phi_{t}$ is defined on all of $X_{s}$ and it is a diffeomorphism from $X_{s}$ to $X_{s-t}$.

(b) Where defined, the flow $\phi_{t}$ preserves the symplectic structures, i.e., if $x \in X_{z} \cap(X \backslash Z)$ is a point where $\phi_{t}(x)$ is defined, then $\phi_{t}^{*}\left(\omega_{z-t}\right)_{\phi_{t}(x)}=\left(\omega_{z}\right)_{x}$. In particular, for $s>t>0$, the map $\phi_{t}$ is a symplectomorphism between $X_{s}$ and $X_{s-t}$.

(c) For $s=t$, there exists an open dense subset $U_{t}=U_{s}$ of $X_{t}$ and an open dense subset $U_{0} \subset X_{0}$ in the smooth locus of $X_{0}$ such that $\phi_{t}$ is a symplectomorphism from $U_{t}$ to $U_{0}$. Moreover, $\phi_{t}$ extends continuously to a map $\phi_{t}: X_{t} \rightarrow X_{0}$.

Using the gradient-Hamiltonian flows, for each $0<t \leq 1$ we construct an integrable $\mu_{t}: X_{t} \rightarrow \mathbb{R}^{n}$ by pulling back the standard integrable system $\mu_{0}: X_{0} \rightarrow \mathbb{R}^{n}$ (arising from the structure of $X_{0}$ as a toric variety) on $X_{0}$ through the maps $\phi_{t}[8$, Theorem 5.2]. More precisely, we define

$$
\mu_{t}:=\phi_{t}^{*} \mu_{0}: X_{t} \rightarrow \mathbb{R}^{n} .
$$

As a result, the moment map image $\mu_{t}\left(X_{t}\right)$ for each $0<t \leq 1$ is equal to the moment map image $\Delta_{0}:=$ $\mu_{0}\left(X_{0}\right)$ of the toric variety $X_{0}$. In particular, we have an integrable system $\mu: X \rightarrow \mathbb{R}^{n}$ on $X \cong X_{1}$ whose image is $\Delta_{0}$. For further details we refer to [8]. 
In what follows it will sometimes be useful to refer to the families of $X_{t}$ and $U_{t}$ with $t \in[0,1]$, and so we define

$$
\begin{aligned}
& \mathcal{X}_{[0,1]}:=\pi^{-1}([0,1]) \subset X \\
& \mathcal{U}_{[0,1]}:=\left\{x \in \mathcal{X}_{[0,1]} \mid x \in U_{\pi(x)}\right\} .
\end{aligned}
$$

3.2. The varying complex structure. We now define a family $\left\{J_{s, t}\right\}$ of complex structures on $X \cong X_{1}$ where $s, t$ are real parameters with $0 \leq s<\infty$ and $0<t \leq 1$. In Section 4 we will choose an appropriate continuous function $t=t(s)$ of $s$ and thus define a 1-parameter family $\left\{J_{s}=J_{s, t(s)}\right\}$ of complex structures which will satisfy the properties asserted in our Theorem 2.3 .

For details we refer to [3,7] but we briefly set some notation. Recall that $\mathcal{P} \cong \mathbb{P}^{N}$ is a standard projective space. By slight abuse of notation we denote also by $\mathcal{P}$ the underlying smooth manifold. Since the usual projective space $\mathbb{P}^{N}$ is naturally a Kähler manifold with Kähler structure $\left(\mathcal{P}, \omega_{\mathcal{P}}, J_{\mathcal{P}}\right)$, we may consider $\mathcal{P}$ as a symplectic manifold $\left(\mathcal{P}, \omega_{\mathcal{P}}\right)$ or as a complex manifold $\left(\mathcal{P}, J_{\mathcal{P}}\right)$. In [3] the authors construct a family of diffeomorphisms

$$
\chi_{s}: \mathcal{P} \rightarrow \mathcal{P}
$$

for $s \in \mathbb{R}$ with $0 \leq s<\infty$ which satisfy the following:

$(\chi-1) \chi_{0}: \mathcal{P} \rightarrow \mathcal{P}$ is the identity function, and

$(\chi-2)$ for any $s$ with $0 \leq s<\infty$, the triple $\left(\mathcal{P}, \omega_{\mathcal{P}}, \chi_{s}^{*}\left(J_{\mathcal{P}}\right)\right)$ is a Kähler structure on $\mathcal{P}$.

The family $\left\{\chi_{s}\right\}$ in (3.5) satisying $(\chi-1)$ and $(\chi-2)$ is not uniquely determined; the general construction given in [3] could yield many such choices of $\left\{\chi_{s}\right\}$. We interpret the diffeomorphisms $\chi_{s}$ as giving rise to aneparameter family of Kähler structures on $\mathcal{P}$ with respect to the same symplectic structure but with varying complex structure.

In this paper, we wish to use the varying complex structures $\chi_{s}^{*} J_{\mathcal{P}}$ on $\mathcal{P}$ to define a family of complex structures on $X$. However, this is not completely straightforward because a smooth submanifold $X$ of $\mathcal{P}$ may be a complex submanifold of $\mathcal{P}$ for the original complex structure $J_{\mathcal{P}}$ but may not be a complex submanifold of $\mathcal{P}$ equipped with the altered complex structure $\chi_{s}^{*} J_{\mathcal{P}}$. To address this issue, the first author and Konno prove the following [7, Proposition 6.1].

Proposition 3.2. ( [7. Proposition 6.1]) Let $V$ be a smooth submanifold of $\mathcal{P}$ with associated embedding $\rho: V \hookrightarrow \mathcal{P}$. Assume that $V$ is a complex submanifold of $\mathcal{P}$ with respect to the complex structure $J_{\mathcal{P}}$ and let $\omega_{V}:=\rho^{*}\left(\omega_{\mathcal{P}}\right)$ denote the corresponding Kähler form on $V$. Let $\chi_{s}: \mathcal{P} \rightarrow \mathcal{P}$ for s a real parameter, $0 \leq s<\infty$, be a family of diffeomorphisms as in (3.5) satisfying $(\chi-1)$ and $(\chi-2)$. Then there exists a family $\left\{\rho_{s}\right\}_{0 \leq s<\infty}$ of embeddings $\rho_{s}: V \hookrightarrow \mathcal{P}$ such that

(a) for all $s$ with $0 \leq s<\infty$ we have $\left.\rho_{s}^{*} \omega_{\mathcal{P}}\right|_{V}=\omega_{V}$,

(b) for all $s$ with $0 \leq s<\infty$ the image $\rho_{s}(V) \subset \mathcal{P}$ is a complex submanifold of $\left(\mathcal{P}, \chi_{s}^{*}\left(J_{\mathcal{P}}\right)\right)$, and

(c) $\rho_{0}=\rho$.

In particular, for any s with $0 \leq s<\infty$ the pair $\left(\omega_{V}, \rho_{s}^{*}\left(\left.\chi_{s}^{*}\left(J_{\mathcal{P}}\right)\right|_{\rho_{s}(V)}\right)\right)$ is a Kähler structure on $V$. Furthermore, for each choice of family $\left\{\chi_{s}\right\}$ as in (3.5), the family of embeddings $\left\{\rho_{s}\right\}$ satisfying the above conditions is unique.

Each fiber $X_{t}$ (for $0<t \leq 1$ ) of our family $X$ is a complex submanifold of $\mathcal{P}$ with respect to the original complex structure $J_{\mathcal{P}}$. Hence, applying Proposition 3.2 to each $X_{t}$, we obtain embeddings

$$
\rho_{s, t}: X_{t} \rightarrow \mathcal{P}
$$


where $s, t$ are real parameters with $0 \leq s<\infty$ and $0<t \leq 1$. We can now define a family $\left\{J_{s, t}\right\}$ of complex structures on $X=X_{1}$. We have the following key diagram (note that it is not commutative):

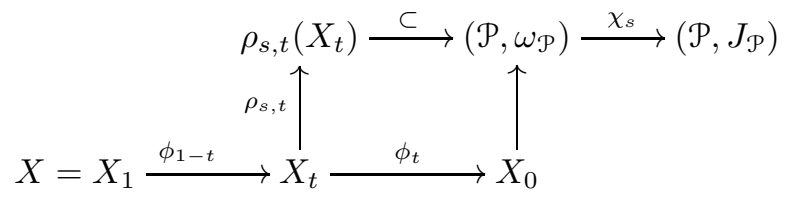

and we have the following.

Definition 3.3. Let $s, t \in \mathbb{R}$ with $0 \leq s<\infty$ and $0<t \leq 1$. Let $\phi_{1-t}: X \cong X_{1} \rightarrow X_{t}$ be the gradientHamiltonian flow and let $\left\{\chi_{s}\right\}$ be a choice of diffeomorphisms as in (3.5) and $\rho_{s, t}: X_{t} \hookrightarrow \mathcal{P}$ be the corresponding embeddings in (3.6). The complex structure $J_{s, t}$ on $X$ is then defined by

$$
J_{s, t} ;=\left(\rho_{s, t} \circ \phi_{1-t}\right)^{*}\left(\left.\chi_{s}^{*} J_{\mathcal{P}}\right|_{\rho_{s, t}\left(X_{t}\right)}\right)
$$

Equivalently, $J_{s, t}$ is the pullback $\left(\chi_{s} \circ \rho_{s, t} \circ \phi_{1-t}\right)^{*} J_{\mathcal{P}}$.

Since both $\rho_{s, t}$ and $\phi_{1-t}$ behave well with respect to the symplectic structures (Propositions 3.1(b) and 3.2(a)), the following is immediate.

Lemma 3.4. Let $s, t \in \mathbb{R}$ with $0 \leq s<\infty$ and $0<t \leq 1$. Then the triple $\left(X \cong X_{1}, \omega=\omega_{1}, J_{s, t}\right)$ is Kähler.

In what follows, we will need two further properties of these embeddings $\rho_{s, t}$ and complex structures $J_{s, t}$, the first of which requires some additional hypotheses on the family $\left\{\chi_{s}\right\}$, as we now explain. As mentioned above, the family $\left\{\chi_{s}\right\}$ given in (3.5) is not unique. However, when $\mathcal{P}$ is equipped with a complex torus action and $V$ happens to be the closure in $\mathcal{P}$ of a torus orbit, then it turns out that the family $\left\{\chi_{s}\right\}$ can be chosen in such a way that $V$ remains a complex submanifold for all of the complex structures $\chi_{s}^{*} J_{\mathcal{P}}$, not just the original complex structure $J_{\mathcal{P}}$. Before proceeding it should be noted that although the statement of Proposition 3.2 (equivalently [7. Proposition 6.5]) contains the hypothesis that $V$ is smooth, it is shown in the proof of [7, Proposition 6.5] that the argument for [7. Proposition 6.1] can be extended in this special case to give a well-defined embedding $\rho$ of $V$, with analogous properties.

Proposition 3.5. Let $\mathbb{H} \subset \mathbb{T}_{\mathcal{P}}$ be a complex subtorus, acting on $\mathcal{P}$ by restriction of the standard $\mathbb{T}_{\mathcal{P}}$-action on $\mathcal{P}$. Let $V$ denote the (possibly singular) closure in $\mathcal{P}$ of the $\mathbb{H}$-orbit of $[1: 1: \cdots: 1] \in \mathcal{P}$. Then there exists a choice of a family $\left\{\chi_{s}\right\}$ as in (3.5), satisfying the assumptions $(\chi-1)$ and $(\chi-2)$, such that for all $s$ with $0 \leq s<\infty$ we have $\rho_{s}=\rho_{0}$, where $\rho_{s}$ is the (unique) embedding associated to $\chi_{s}$ constructed in Proposition 3.2 above. In particular, on the smooth locus of $V$, the complex structures $\chi_{s}^{*} J_{\mathcal{P}}$ and $J_{\mathcal{P}}$ agree, for all $s$.

Since the technical aspects of the construction of the above family $\left\{\chi_{s}\right\}$ are not used in this manuscript, we do not discuss it further here; for details see [37]. In the setting of this manuscript, the fiber $X_{0}:=\pi^{-1}(0)$ over 0 of our family $X$ is by assumption the closure of the $\mathbb{T}_{0}$-orbit of $[1: 1: \cdots: 1]$ for $\mathbb{T}_{0} \subset \mathbb{T}_{\mathcal{P}}$ a subtorus of $\mathbb{T}_{\mathcal{P}}$. Hence Proposition 3.5 applies, and we therefore obtain a family $\left\{\chi_{s}\right\}$ of diffeomorphisms as in (3.5) (satisfying the assumptions $(\chi-1)$ and $(\chi-2)$ ) such that the associated $\rho_{s}$ 's leave $X_{0}$ invariant, i.e., $\rho_{s}=\rho_{0}$ on $X_{0}$ for all $0 \leq s<\infty$. This will be crucial in what follows so we now record, by way of emphasis, that

\section{henceforth, we assume that the family $\left\{\chi_{s}\right\}$ is chosen in such a way that the conclusion of Proposition 3.5 holds.}

Given this choice of $\left\{\chi_{s}\right\}$, in our later arguments we need to know that the maps $\rho_{s, t}$ defined in (3.6) satisfy some continuity conditions with respect to the parameter $t$. We record the following.

Proposition 3.6. Let $s \in \mathbb{R}$ with $0 \leq s<\infty$. Let $X \subseteq \mathcal{P} \times \mathbb{C}$ be the toric degeneration as above and $\left\{\chi_{s}\right\}$ be a family of diffeomorphisms as above, chosen so that the conclusion of Proposition 3.5 holds. Then

(1) the map $X_{[0,1]} \rightarrow \mathcal{P}$ given by $x \mapsto \rho_{s, \pi(x)}(x)$ is continuous, and 
(2) the map $\mathfrak{U}_{[0,1]} \rightarrow \mathcal{P} \times \mathbb{C}$ given by $x \mapsto\left(\rho_{s, \pi(x)}(x), \pi(x)\right)$ is a diffeomorphism onto its image.

Proof. We first review the construction of the map $\rho_{s}$ from [7]. Let $\chi_{s}: \mathcal{P} \rightarrow \mathcal{P}$ be the diffeomorphisms from (3.5). Let $\psi_{s}=\chi_{0} \circ \chi_{s}^{-1}: \mathcal{P} \rightarrow \mathcal{P}$ and let $\omega_{s}=\left(\chi_{s}^{-1}\right)^{*} \omega_{\mathcal{P}}$. By property $(\chi-2)$ of the family $\left\{\chi_{s}\right\}$ we know that $\left(\omega_{s}, J_{\mathcal{P}}\right)$ is a Kähler structure on $\mathcal{P}$. Thus, any submanifold $V$ of $\mathcal{P}$ which is complex with respect to $J_{\mathcal{P}}$ is also a symplectic submanifold with respect to $\omega_{s}$. Hence we can define a time-dependent vector field $\mathbb{V}_{s}$ on $\mathcal{P}$ by

$$
\left(\mathbb{V}_{s}\right)_{\psi_{s}(p)}=\left.\frac{d}{d \tau} \psi_{s+\tau}(p)\right|_{\tau=0}
$$

for $p \in \mathcal{P}$. Following [7] we further define a vector field $Y_{s}$ on $V$ by

$$
\iota_{Y_{s}}\left(\left.\omega_{s}\right|_{V}\right)=-\left.\psi_{s}^{*}\left(\iota_{\mathbb{V}_{s}} \omega_{\mathcal{P}}\right)\right|_{V} .
$$

Letting $\varphi_{s}$ denote the corresponding flow of the vector field $Y_{s}$, again following [7] we finally define $\rho_{s}$ by

$$
\rho_{s}:=\left.\chi_{s}^{-1} \circ \rho_{0} \circ \varphi_{s} \circ \chi_{0}\right|_{V} .
$$

The construction just recounted deals with a single submanifold $V \subseteq \mathcal{P}$. To prove the proposition we must show that this construction can be extended to one on a family $X \subseteq \mathcal{P} \times \mathbb{C}$ in a way which guarantees the claimed smoothness and continuity properties with respect to the extra parameter. To do this, we first define $\widehat{\chi}_{s}: \mathcal{P} \times \mathbb{C} \rightarrow \mathcal{P} \times \mathbb{C}$ by $\hat{\chi}_{s}(x, t)=\left(\chi_{s}(x), t\right)$ for $(x, t) \in \mathcal{P} \times \mathbb{C}$ and $\widehat{\psi}_{s}:=\widehat{\chi}_{0} \circ \widehat{\chi}_{s}^{-1}$. We then define a time-dependent vector field $\widehat{\mathbb{V}}_{s}$ on $\mathcal{P} \times \mathbb{C}$ by

$$
\left(\widehat{\mathbb{V}}_{s}\right)_{\left(\psi_{s}(p), t\right)}:=\left.\frac{d}{d \tau} \widehat{\psi}_{s+\tau}(p, t)\right|_{\tau=0}
$$

and a vector field $\widehat{\mathbb{Y}}_{s}$ on $X_{\text {smooth }}$, the smooth locus of $X$, by

$$
\iota_{\widehat{\mathbb{Y}}_{s}}\left(\widehat{\omega}_{s}\right)=\iota_{\widehat{\mathbb{Y}}_{s}}\left(\left(\widehat{\chi}_{s}^{-1}\right)^{*} \Omega\right)=-\left.\widehat{\psi}_{s}^{*}\left(\iota_{\widehat{\mathbb{V}}_{s}} \Omega\right)\right|_{x_{s m o o t h}}
$$

where $\Omega$ is the product Kähler structure on $\mathcal{P} \times \mathbb{C}$. Note that $\widehat{\mathbb{V}}_{s}=\left(\mathbb{V}_{s}, 0\right)$ by definition of $\widehat{\psi}_{s}$ and by construction $\widehat{\mathbb{Y}}_{s}$ is a smooth vector field on $X_{\text {smooth }}$. We wish to analyze the relation between $Y_{s}$, defined via the above construction from [7] on each $X_{t}$ separately, and $\widehat{\mathbb{Y}}_{s}$, for which we need some preliminaries. Let $p r_{1}: \mathcal{P} \times \mathbb{C} \rightarrow \mathcal{P}$ be the projection to the first factor and $\mathcal{V} \subseteq T \mathcal{X}_{\text {smooth }}$ denote the vertical subbundle of $T \mathcal{X}_{\text {smooth }}$ with respect to $p r_{1}$, i.e., $\mathcal{V}_{x}:=\operatorname{ker}\left(d\left(p r_{1}\right)_{x}\right)$ for each $x \in X_{\text {smooth }}$. Then $\mathcal{V}$ is a smooth symplectic subbundle of $T X_{\text {smooth }}$ with respect to $\left.\Omega\right|_{x_{\text {smooth }}}$, so there is a canonical decomposition $T X_{\text {smooth }} \cong \mathcal{V} \oplus \mathcal{V}^{\Omega}$ and the projection $T X_{\text {smooth }} \rightarrow \mathcal{V}$ is smooth.

We now claim that

$$
-\widehat{\psi}_{s}^{*}\left(\iota_{\widehat{\mathbb{V}}_{s}} \Omega\right)=p r_{1}^{*}\left(-\Psi_{s}^{*}\left(\iota_{\mathbb{V}_{s}} \omega_{\mathcal{P}}\right)\right)
$$

as 1 -forms on $\mathcal{P} \times \mathbb{C}$. Indeed, for any $w \in T(\mathcal{P} \times \mathbb{C}) \cong T \mathcal{P} \oplus T \mathbb{C}$, we may decompose $w=\left(w_{\mathcal{P}}, w_{\mathbb{C}}\right)$ into its two factors and compute

$$
\begin{aligned}
\widehat{\psi}_{s}^{*}\left(\iota_{\widehat{\mathbb{V}}_{s}} \Omega\right)(w) & =\Omega\left(\widehat{\mathbb{V}}_{s},\left(\widehat{\psi}_{s}\right)_{*}(w)\right) \\
& =\omega_{\mathcal{P}}\left(\mathbb{V}_{s},\left(\left(\widehat{\psi}_{s}\right)_{*} w\right)_{\mathcal{P}}\right) \text { since } \widehat{\mathbb{V}}_{s}=\left(\mathbb{V}_{s}, 0\right) \\
& =\omega_{\mathcal{P}}\left(\mathbb{V}_{s},\left(\Psi_{s}\right)_{*}\left(\omega_{\mathcal{P}}\right)\right) \text { since } \widehat{\psi}_{s} \text { acts as the identity on the } \mathbb{C} \text { factor } \\
& =\left(\Psi_{s}\right)_{*}\left(\iota \mathbb{V}_{s} \omega_{\mathcal{P}}\right)\left(w_{\mathcal{P}}\right) \\
& =p r_{1}^{*}\left(\Psi_{s}^{*}\left(\iota_{\mathbb{V}_{s}} \omega_{\mathcal{P}}\right)\right)(w) .
\end{aligned}
$$


Now suppose $Z \in \mathcal{V} \subseteq T X_{\text {smooth }}$, so $Z=\left(Z_{\mathcal{P}}, 0\right)$ where $Z_{\mathcal{P}} \in T \mathcal{P} \cap T X_{\text {smooth }}$. We have

$$
\begin{aligned}
\iota_{\widehat{\mathbb{Y}}_{s}}\left(\widehat{\omega}_{s}\right)(Z) & =-\widehat{\psi}_{s}^{*}\left(\iota_{\widehat{\mathbb{V}}_{s}} \Omega\right)(Z) \\
& =\operatorname{pr}_{1}^{*}\left(-\Psi_{s}^{*}\left(\iota_{\mathbb{V}_{s}} \omega_{\mathcal{P}}\right)\right)(Z) \\
& =-\Psi_{s}^{*}\left(\iota_{\mathbb{V}_{s}} \omega_{\mathcal{P}}\right)\left(Z_{\mathcal{P}}\right) \\
& =\iota_{Y_{s}}\left(\omega_{s}\right)\left(Z_{\mathcal{P}}\right)
\end{aligned}
$$

where the first equality is by (3.10), the second by (3.11), and the last is the definition (3.9) of $Y_{s}$ on each fiber. From this it follows that the symplectic-orthogonal projection $\left(\widehat{Y}_{s}\right)_{\text {vert }}$ of $\widehat{Y}_{s}$ to the vertical subbundle $\nu \subseteq T X_{\text {smooth }}$ agrees, fiberwise, with the vector field $Y_{s}$ defined using the original construction from [7]. Since the symplectic-orthogonal projection is smooth, as argued above, it follows that the vector field $Y_{s}$, considered together on all of $X_{\text {smooth }}$, is smooth on $X_{\text {smooth }}$. Let $\widehat{\varphi}_{s}$ denote the flow corresponding to $\left(\widehat{Y}_{s}\right)_{\text {vert }}$, which exists since it exists fiberwise for each $X_{t}$ with $t \neq 0$, and for $X_{0}$, the argument in the proof of [7. Proposition 6.1] shows that a flow exists and extends continuously to all of $X_{0}$. Hence the statement (1) of the proposition now follows.

To prove (2), we first observe that the above argument shows that the map $\mathfrak{U}_{[0,1]} \rightarrow \mathcal{P} \times \mathbb{C}$ given by $x \mapsto\left(\rho_{s, \pi(x)}(x), \pi(x)\right)$ is smooth. So it suffices to show that this map is smoothly invertible. We know that for each fixed $t \in \mathbb{C}$ with $t \neq 0$, the map $\rho_{s, t}: X_{t} \rightarrow \mathcal{P}$ is an embedding. Moreover, Proposition 3.5]implies that on $U_{0} \subseteq X_{0}$ we have $\rho_{s, 0}=\rho_{0}$, hence $\rho_{s, 0}$ is also an embedding. It follows that $x \mapsto\left(\rho_{s, \pi(x)}(x), \pi(x)\right)$ is injective, so it is bijective on its image. It remains to show that the inverse map is also smooth. Since $\mathcal{U}_{[0,1]}$ lies in $X_{\text {smooth }}$ we may decompose $T \mathcal{U}_{[0,1]}$ into the vertical subbundle $\mathcal{V}$ and its complement $\mathcal{V}^{\Omega}$. With respect to this decomposition and the standard decomposition $T \mathcal{P} \oplus T \mathbb{C}$ of $\mathcal{P} \times \mathbb{C}$, the derivative of the above map at a point in $\pi^{-1}(t)$ is of the form

$$
\left[\begin{array}{cc}
\left(\rho_{s, t}\right)_{*} & \star \\
0 & I
\end{array}\right]
$$

where $I$ is an isomorphism and $\left(\rho_{s, t}\right)_{*}$ is injective. Thus the whole derivative is also injective, and it follows that the inverse mapping is smooth.

3.3. Pullbacks of prequantum data. The main result of this manuscript deals with quantizations, and in particular with sections of certain prequantum line bundles. In this section, we show that the gradientHamiltonian flows $\phi_{1-t}$ and the embeddings $\rho_{s, t}$ from Section 3.2 lift to the total spaces of the relevant line bundles. This will be crucial for our constructions below. Recall that we have the prequantum data $\left(L_{\mathcal{P}}, \nabla_{\mathcal{P}}, h_{\mathcal{P}}\right)$ and $\left(L_{X}, \nabla_{X}, h_{X}\right)$ respectively on $\mathcal{P}$ and $X$ and that the latter restricts to give prequantum data on the fibers $X_{t}$, which we denote by $\left(L_{t}, \nabla_{t}, h_{t}\right)$.

We first recall that the horizontal lift of the gradient-Hamiltonian flow with respect to the connection $\nabla x$ preserves the connections and Hermitian metrics on each fiber [7, Proposition 4.3].

Lemma 3.7. ( [7, Proposition 4.3])

Let $s, t \in \mathbb{R}$ with $s \geq t>0$.

(1) If $s>t$, there exists a unique horizontal lift $\tilde{\phi}_{t}: L_{s} \rightarrow L_{s-t}$ of the gradient-Hamiltonian flow $\phi_{t}: X_{s} \rightarrow$ $X_{s-t}$ to the total spaces of the prequantum line bundles. The lift $\tilde{\phi}_{t}$ is an isomorphism of line bundles and also preserves the fiberwise connections and Hermitian structures, i.e., $\tilde{\phi}_{t}^{*} \nabla_{s-t}=\nabla_{s}$ and $\tilde{\phi}_{t}^{*} h_{s-t}=h_{s}$.

(2) If $s=t$, there there exists a unique horizontal lift $\tilde{\phi}_{s=t}:\left.\left.L_{t}\right|_{U_{t}} \rightarrow L_{0}\right|_{U_{0}}$ of the gradient-Hamiltonian flow $\phi_{t}: U_{t} \rightarrow U_{0}$ to the total spaces of the (restricted) prequantum line bundles. The lift $\tilde{\phi}_{t}$ is an isomorphism of line bundles and also preserves the fiberwise connections and Hermitian structures, i.e., $\tilde{\phi}_{t}^{*} \nabla_{0}=\nabla_{t}$ and $\tilde{\phi}_{t}^{*} h_{0}=h_{t}$ (restricted to $U_{t}$ and $\left.U_{0}\right)$.

(3) The map $\left.L_{0}\right|_{U_{0}} \times[0,1] \rightarrow L_{x}$ given by $(x, t) \mapsto \tilde{\phi}_{t}^{-1}(x)$ is smooth, where the domain $\left.L_{0}\right|_{U_{0}} \times[0,1]$ is given the standard smooth structure induced from the product structure. 
Proof. The argument is essentially the same as in [7]. For the statement in (3) we note that the gradientHamiltonian flow on $X \backslash Z$ is smooth and hence its horizontal lift is also smooth. Now an argument similar to that in the proof of Proposition 3.6yields the result.

We remark that it follows from the above lemma that the following diagram commutes for $t$ with $0<$ $t<1$ :

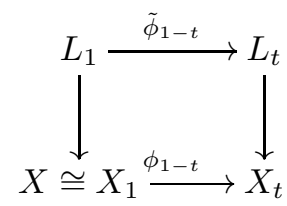

We will also need a similar statement for the embeddings $\rho_{s, t}$ [7, Proposition6.3(1)].

Lemma 3.8. ( [7. Proposition 6.3(1)]) There exists a lift $\tilde{\rho}_{s, t}:\left.L_{t} \rightarrow L_{\mathcal{P}}\right|_{\rho_{s, t}\left(X_{t}\right)}$ of $\rho_{s, t}$ to the total spaces of the line bundles $L_{t}$ and $\left.L_{\mathcal{P}}\right|_{\rho_{s, t}\left(X_{t}\right)}$ which identifies the prequantum data. In particular, the following diagram commutes:

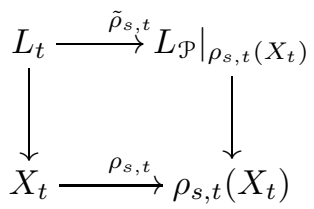

We need a smoothness property for the map $\widetilde{\rho}_{s, t}$, analogous to Proposition 3.6 for the $\rho_{s, t}$.

Proposition 3.9. Let $s \in \mathbb{R}, 0 \leq s<\infty$ be fixed. Let $X \subseteq \mathcal{P} \times \mathbb{C}$ be the toric degeneration as above and $\left\{\chi_{s}\right\}$ be the family of diffeomorphisms as above (in particular chosen so that the conclusion of Proposition 3.5 holds). For any $0 \leq t \leq 1$, let $\rho_{s, t}: X_{t} \hookrightarrow \mathcal{P}$ be the embedding defined in Proposition 3.2 and let $\tilde{\rho}_{s, t}$ be the lifting of $\rho_{s, t}$ as defined in Lemma 3.8 Let $L_{\mathcal{U}}$ denote the restriction of the line bundle $L_{X}$ to the open subset $\mathcal{U}_{[0,1]}$ defined in (3.4). Then the map

$$
\tilde{\rho}_{s}: L_{\mathcal{U}} \rightarrow L_{\mathcal{P}} \times \mathbb{C}, \quad(x, \xi) \mapsto\left(\tilde{\rho}_{s, \pi(x)}(\xi), \pi(x)\right)
$$

where the pair $(x, \xi)$ consists of a point $x \in \mathcal{U}$ and $\xi \in L_{x}$, is a diffeomorphism onto its image.

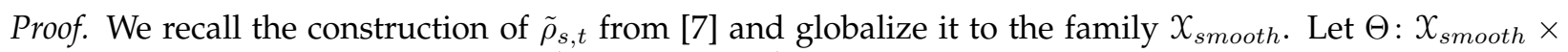
$[0, \infty) \rightarrow \mathcal{P} \times \mathbb{C}$ be the map $(x, s) \mapsto\left(\rho_{s, \pi(x)}(x), \pi(x)\right)$. Let $\left(L^{\prime}, \nabla^{\prime}, h^{\prime}\right)=\Theta^{*}\left(L_{\mathcal{P} \times \mathbb{C}}, \nabla_{\mathcal{P} \times \mathbb{C}}, h_{\mathcal{P} \times \mathbb{C}}\right)$. Note that $\left.L^{\prime}\right|_{\text {smooth }} \times\left\{s_{0}\right\}=\rho_{s_{0}}^{*} L_{X}$.

Let $Z \in \operatorname{Vect}\left(L^{\prime}\right)$ be the horizontal lift to $L^{\prime}$ with respect to $\nabla^{\prime}$ of the vector field $\frac{\partial}{\partial s}$ on $X_{\text {smooth }} \times[0, \infty)$. Then the flow of $z$ through time $s$ induces a diffomorphism between the bundles $\rho_{0}^{*} L_{x}$ and $\rho_{s}^{*} L_{x}$ over $X_{\text {smooth }} \times\{0\}$ and $X_{\text {smooth }} \times\{s\}$. This is the same as a diffeomorphism $L x \rightarrow L_{\rho_{s_{0}}(X)}$ lifting the map $\rho_{s_{0}}$, and we denote it by $\tilde{\rho}_{s}$. Since $\mathcal{U}_{[0,1]}$ is a subset of $X_{\text {smooth }}$ by construction, the map $\tilde{\rho}_{s}$ is defined on $L_{U}$, and is a diffeomorphism onto its image.

If we restrict the map $\Theta$ to the (smooth locus in the) fibre $X_{t} \times[0, \infty)$ we obtain a map $\Theta_{t}:(x, s) \mapsto \rho_{s, t}(x)$, which agrees with the map used in [7. Claim 6.4] to construct the lift of $\rho_{s, t}$ over the submanifold $X_{t}$. Furthermore, it is clear that $\Theta_{t}^{*}\left(L_{\mathcal{P} \times \mathbb{C}}, \nabla_{\mathcal{P} \times \mathbb{C}}, h_{\mathcal{P} \times \mathbb{C}}\right)$ restricts to $\left(L^{\prime}, \nabla^{\prime}, h^{\prime}\right)$ on $X_{t} \times[0, \infty)$; this agrees with the data used in the construction in [7]. Therefore the lifting $\tilde{\rho}_{s}$ constructed above agrees on $U_{t}$ with the map $\tilde{\rho}_{s, t}$ as constructed in [7], and the formula given in (3.12) agrees with the map $\tilde{\rho}_{s}$ constructed in the previous paragraph.

Finally, we analyze the behavior of the diffeomorphisms $\chi_{s}: \mathcal{P} \rightarrow \mathcal{P}$ of (3.5) with respect to the prequantum data. Recall that $L_{\mathcal{P}}$ is a holomorphic line bundle with respect to the canonical complex structure $J_{\mathcal{P}}$ on 
$\mathcal{P}$ (i.e. its transition functions are holomorphic), and hence there exists a differential operator $\bar{\partial}$ defining the space of holomorphic sections $H^{0}\left(\mathcal{P}, L_{\mathcal{P}}, \bar{\partial}\right)$ of $L_{\mathcal{P}}$ over $\left(\mathcal{P}, J_{\mathcal{P}}\right)$. We recall the following [7, Theorem 5.3(A)].

Lemma 3.10. ( [7. Theorem 5.3(A)]) There exists a lift $\tilde{\chi}_{s}$ of $\chi_{s}$ to an isomorphism of the line bundle $L_{\mathcal{P}}$ such that the following diagram commutes

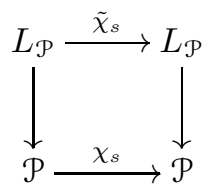

and such that the connection $\nabla_{\mathcal{P}}$ is the canonical Chern connection for the Hermitian holomorphic line bundle $\left(L_{\mathcal{P}}, h_{\mathcal{P}}, \tilde{\chi}_{s}^{*} \bar{\partial}\right)$.

From now on we notate

$$
\bar{\partial}_{s}:=\tilde{\chi}_{s}^{*}(\bar{\partial})
$$

and denote by $H^{0}\left(\mathcal{P}, L_{\mathcal{P}}, \bar{\partial}_{s}\right)$ the corresponding space of sections. From the definitions of the respective holomorphic structures, it is immediate that the pullback by $\tilde{\chi}_{s}^{*}$ of a section which is holomorphic with respect to $\bar{\partial}$ is holomorphic with respect to $\bar{\partial}_{s}$.

Lemma 3.11. The pullback $\tilde{\chi}_{s}^{*}(\sigma)$ of a section $\sigma \in H^{0}\left(\mathcal{P}, L_{\mathcal{P}}, \bar{\partial}\right)$ is an element of $H^{0}\left(\mathcal{P}, L_{\mathcal{P}}, \bar{\partial}_{s}\right)$.

In fact, in our arguments below we will need to pull back sections to the original variety $X$ via the diagram

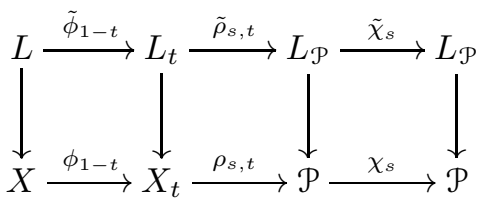

obtained by composing the three diagrams above. We record the following.

Lemma 3.12. Let $s, t \in \mathbb{R}$ with $0 \leq s<\infty$ and $0 \leq t<1$. Following notation as above, the pullbacks $\tilde{\chi}_{s}^{*}, \tilde{\rho}_{s, t}^{*}$ and $\tilde{\phi}_{1-t}^{*}$ preserve holomorphic sections, so in particular there is a well-defined map

$$
\tilde{\phi}_{1-t}^{*} \circ \tilde{\rho}_{s, t}^{*} \circ \tilde{\chi}_{s}^{*}: H^{0}\left(\mathcal{P}, L_{\mathcal{P}}, \bar{\partial}_{0}\right) \rightarrow H^{0}\left(X, L, \bar{\partial}_{s, t}\right)
$$

where $\bar{\partial}_{0}$ denotes the standard holomorphic structure on $\left(\mathcal{P}=\mathbb{P}^{N}, \omega_{\mathcal{P}}, J_{\mathcal{P}}\right)$.

Proof. The differential operator $\bar{\partial}_{s, t}$ is associated to the complex structure $\tilde{\phi}_{1-t}^{*} \tilde{\rho}_{s, t}^{*} \tilde{\chi}_{s}^{*}\left(J_{\mathcal{P}}\right)$ obtained by pullback, so the result is immediate from the definitions.

We also record the important fact that the Bohr-Sommerfeld fibres of the integrable system on $X$ correspond to those on $X_{0}$. In particular, the Bohr-Sommerfeld set is the set $W_{0}$ defined in (2.1).

Proposition 3.13. Let $W_{0}:=\iota^{*}\left(\Delta_{\mathcal{P}} \cap \mathbb{Z}^{N}\right) \subseteq \Delta_{0} \cap \mathbb{Z}^{n}$ as defined in (2.1). Then the Bohr-Sommerfeld fibres in $X$ are precisely the preimages of points in $W_{0}$ under the integrable system $\mu$ constructed in (3.3).

Proof. Since the gradient-Hamiltonian flow lifts to the line bundle preserving the connection, it follows that the gradient-Hamiltonian flow maps Bohr-Sommerfeld fibres in $X$ to Bohr-Sommerfeld fibres in $X_{0}$. Since the Bohr-Sommerfeld fibres of the torus moment map are the preimages of $W_{0}$, and the integrable system $\mu: X \rightarrow \mathbb{R}^{n}$ was constructed by pulling back the moment map for the torus action on $X_{0}$, we obtain the result. 
3.4. Varying bases of sections $\left\{\sigma_{s, t}^{m}\right\}$ of $H^{0}\left(X, L, \bar{\partial}_{s, t}\right)$. Our main result, Theorem 2.3, asserts the existence of a basis of sections $\left\{\sigma_{s}^{m}\right\}$, indexed by $m \in W_{0}$ and dependent on a real parameter $s$, where each $\sigma_{s}^{m}$ is holomorphic with respect to the complex structure $J_{s}$. In this section, we take a step in this direction by using the results of Section 3.3 to define sections $\sigma_{s, t}^{m}$ in $H^{0}\left(X, L, \bar{\partial}_{s, t}\right)$.

The sections $\sigma_{s, t}^{m}$ are constructed using certain standard sections of $\left(\mathcal{P}, L_{\mathcal{P}}\right)$ which we now recall. It is well-known that $\mathcal{P}$ is a toric variety with respect to the standard action of its torus $T_{\mathcal{P}}$. For any integer lattice point in $\Delta_{\mathcal{P}} \cap \mathbb{Z}^{N}$, there is a well-known method (see e.g. [6]) to associate to it a holomorphic section in $H^{0}\left(\mathcal{P}, L_{\mathcal{P}}, \bar{\partial}_{0}\right)$. In fact, this association yields a bijective correspondence between $\Delta_{\mathcal{P}} \cap \mathbb{Z}^{N}$ and a basis for $H^{0}\left(\mathcal{P}, L_{\mathcal{P}}, \bar{\partial}_{0}\right)$ which we denote as

$$
\tilde{m} \in \Delta_{\mathcal{P}} \cap \mathbb{Z}^{N} \mapsto \sigma^{\tilde{m}} \in H^{0}\left(\mathcal{P}, L_{\mathcal{P}}, \bar{\partial}_{0}\right) .
$$

Now recall that $W_{0}:=\iota^{*}\left(\Delta_{\mathcal{P}} \cap \mathbb{Z}^{N}\right)$ is defined to be precisely the lattice points in $\Delta_{0}$ which lie in the image of $\iota^{*}$ of $\Delta_{\mathcal{P}} \cap \mathbb{Z}^{N}$. Thus for any $m \in W_{0}$, by assumption there exists a preimage $\tilde{m}$ of $m$ under $\iota^{*}$.

We can now define our set of sections $\sigma_{s, t}^{m}$. The sections depend on a choice of preimage $\tilde{m}$ for each $m \in W_{0}$, but their essential properties - such as those asserted in Theorem 2.3- are independent of these choices. For this reason and for simplicity we suppress this choice from the notation. Specifically, we have the following. (It may be helpful for the reader to refer to the diagram (3.13).)

Definition 3.14. For each $m \in W_{0}$, let $\tilde{m} \in \Delta_{\mathcal{P}} \cap \mathbb{Z}^{N}$ denote a fixed choice of preimage of $m$ under $\iota^{*}$. Let $s, t \in \mathbb{R}$ with $0 \leq s<\infty$ and $0<t \leq 1$. We define

$$
\sigma_{s, t}^{m}:=\tilde{\phi}_{1-t}^{*} \tilde{\rho}_{s, t}^{*} \tilde{\chi}_{s}^{*} \sigma^{\tilde{m}} \in H^{0}\left(X, L, \bar{\partial}_{s, t}\right) .
$$

In the next section we will find an appropriate function $t=t(s)$ so that the bases $\left\{\sigma_{s}^{m}:=\sigma_{s, t=t(s)}^{m}\right\}$ will satisfy the convergence conditions asserted in Theorem 2.3 with respect to the complex structures $J_{s}:=J_{s, t=t(s)}$ and $\bar{\partial}_{s}:=\bar{\partial}_{s, t=t(s)}$.

\section{Proof of THE MAIN THeOREM}

We now proceed to a proof of the main result of this manuscript, Theorem 2.3. Much of this section is devoted to proving results about the sections $\sigma_{s, t}^{m}$ defined in Section 3.4, under certain hypotheses on the parameters $s$ and $t$. At the end of this section we choose an appropriate function $t=t(s)$ so that the sections $\sigma_{s}^{m}:=\sigma_{s, t(s)}^{m}$ depend only on the single parameter $s$ and have the correct convergence properties.

We begin our discussion with a statement about supports. Specifically, part of the assertion of Theorem 2.3 is that a certain (normalized) section weakly converges to a dirac-delta function on the corresponding Bohr-Sommerfeld fiber. In particular, the support of $\sigma_{s, t=t(s)}^{m}$ must concentrate on a neighborhood of the Bohr-Sommerfeld fiber as $s$ gets large. We make this precise in the proposition below, for which we need some preliminaries. Let $m \in W_{0} \subset \mathfrak{t}_{0}^{*}$. For a real number $\eta>0$, let $B_{\eta}(m)$ denote the open ball of radius $\eta$ around $m$ with respect to the usual metric on $\mathfrak{t}_{0}^{*}$. We introduce the following notation:

$$
B_{\eta}(m):=\mu^{-1}\left(B_{\eta}(m)\right), \quad B_{\eta, t}(m):=\mu_{t}^{-1}\left(B_{\eta}(m)\right), \quad B_{\eta, 0}(m):=\mu_{0}^{-1}\left(B_{\eta}(m)\right)
$$

where $\mu, \mu_{t}$ and $\mu_{0}$ are the moment maps for the integrable systems on $X, X_{t}$ and $X_{0}$ respectively. When the point $m$ is clear from context, we sometimes write $B_{\eta, t}=B_{\eta, t}(m)$, etc. We also let $d(v o l)$ denote the symplectic volume form on $X_{t}$ and $\rho_{s, t}\left(X_{t}\right)$ for all $s$ and $t$; since the relevant maps between these spaces preserve symplectic structures, the ambiguity in this notation does not pose problems. We let $|\cdot|$ denote the norm with respect to the hermitian metric on all the line bundles; again, all relevant maps preserve the hermitian metric so there is no ambiguity. We let $\|\cdot\|_{L^{1}(\cdot)}$ denote the $L^{1}$-norm of a section over some space; for the sake of space and readability we will occasionally omit the explicit mention of the space in the notation and write simply $\|\cdot\|$.

We can now state and prove the following. 
Proposition 4.1. Let $m \in W_{0}$ be an interior point and let $\sigma_{s, t}^{m} \in H^{0}\left(X, L, \bar{\partial}_{s, t}\right)$ be the section defined in (3.14). Then there exists a continuous function $t^{\prime}=t^{\prime}(s):[0, \infty) \rightarrow[0,1]$ such that for every $\epsilon>0$ and $\eta>0$, there exists $s_{0}>0$ such that

$$
\int_{X \backslash B_{\eta}}\left|\frac{\sigma_{s, t}^{m}}{\left\|\sigma_{s, t}^{m}\right\|_{L^{1}(X)}}\right| d(v o l)<\epsilon
$$

for all $s>s_{0}$ and $0 \leq t \leq t^{\prime}(s)$, and moreover, $\lim _{s \rightarrow \infty} t^{\prime}(s)=0$.

Remark 4.2. We believe the result of Proposition 4.1 holds for boundary points as well as interior points but we restrict ourselves to interior points for the purposes of this paper.

The proof of Proposition 4.1 requires several steps, the first of which states that the analogous result is true for the special fiber $X_{0}$. We quote the following.

Lemma 4.3. ( [7. Proposition 6.6, (3) and (4)]) Let $m \in W_{0}$ be an interior point and let $\tilde{m} \in \Delta_{\mathcal{P}} \cap \mathbb{Z}^{N}$ denote the preimage of $m$ fixed in Definition 3.14 For any $\epsilon>0$ and $\eta>0$, there exists $s_{0}>0$ such that

$$
\int_{X_{0} \backslash B_{\eta, 0}}\left|\frac{\tilde{\chi}_{s}^{*}\left(\sigma^{\tilde{m}}\right)}{\left\|\tilde{\chi}_{s}^{*}\left(\sigma^{\tilde{m}}\right)\right\|_{L^{1}\left(X_{0}\right)}}\right| d(v o l)<\epsilon
$$

for all $s>s_{0}$.

The following estimate will also be useful. Roughly, it says that for any fixed $s>0$ and any $\epsilon>0$, there are fibers $X_{t}$ of the family which are sufficiently close to $X_{0}$ such that the two maps $\rho_{s, t}$ and $\phi_{t}$ do not differ on $X_{t}$ by more than distance $\epsilon$.

Lemma 4.4. Let $s, t \in \mathbb{R}$ with $0<s<\infty$ and $0 \leq t \leq 1$, let $\rho_{s, t}: X_{t} \rightarrow \mathcal{P}$ be the embedding in Proposition 3.2 . let $\phi_{t}: X_{t} \rightarrow X_{0}$ denote the gradient-Hamiltonian flow, and let $\epsilon>0$. Then there exists $t_{0}>0$ such that for any $x \in X_{t}$ with $0<t<t_{0}$,

$$
d_{\mathcal{P}}\left(\rho_{s, t}(x), \phi_{t}(x)\right)<\epsilon
$$

where $d_{\mathcal{P}}$ denotes the distance function on $\mathcal{P}$ induced from the Kähler metric on $\left(\mathcal{P}, \omega_{\mathcal{P}}, J_{\mathcal{P}}\right)$.

Proof. First, we show that we can choose $t$ small enough that $\phi_{t}(x)$ is close to $x$, uniformly in $X_{t}$. Note that this part of the argument is independent of the parameter $s$. Let

$$
B:=\{(x, t) \in \mathcal{X} \times[0,1] \mid \pi(x) \in[0,1], t \leq \pi(x)\} \subseteq \mathcal{P} \times[0,1] \times[0,1] \subseteq \mathcal{P} \times \mathbb{C} \times[0,1] .
$$

Then $B$ is a closed subset of the compact space $\mathcal{P} \times[0,1] \times[0,1]$, and is therefore compact. Consider the map $\Psi: B \rightarrow B$ given by $\Psi(x, t)=\left(\phi_{t}(x), \pi(x)-t\right)$. Note that $\Psi$ is well-defined since $t \leq \pi(x)$ by assumption so $\pi(x)-t \geq 0$. It follows from [8, Part 1, Theorem 4.1] that $\Psi$ is continuous as a function from $B$ to itself, and hence uniformly continous. In particular, this implies that for any $\delta>0$, there exists a $t_{0}>0$ such that for any $t<t_{0}$ we have $d_{\mathcal{P}}\left(\phi_{t}(x), x\right)<\delta$ for any $x \in X_{t}$.

We next analyze the embeddings $\rho_{s, t}: X_{t} \hookrightarrow \mathcal{P}$. Recall that $X_{[0,1]}$ denotes $\pi^{-1}([0,1]) \subset \mathcal{X}$. For a fixed $s$, let $f_{s}$ denote the map $X_{[0,1]} \rightarrow \mathcal{P}$ given by $x \mapsto \rho_{s, \pi(x)}(x)$. Then $f_{s}$ is continuous by Proposition 3.6, and therefore also uniformly continuous since $X_{[0,1]}$ is compact. Recall from Proposition 3.5 that $\rho_{s, 0}=\mathrm{id}$ for all $s$. Thus for any $x \in X_{t}$ we have

$$
f_{s}\left(\phi_{t}(x)\right)=\rho_{s, 0}\left(\phi_{t}(x)\right)=\phi_{t}(x)
$$

since $\phi_{t}(x) \in X_{0}$. Now let $\epsilon>0$ be given. Choose $\delta>0$ such that $d_{\mathcal{P}}(x, y)<\delta$ implies $d_{\mathcal{P}}\left(f_{s}(x), f_{s}(y)\right)<\epsilon$ for any $x, y \in X_{[0,1]}$. Then choose $t_{0}$ so that $0<t<t_{0}$ implies $d_{\mathcal{P}}\left(\phi_{t}(x), x\right)<\delta$ for all $x \in X_{t}$, as above. Then for all $x \in X_{t}$ with $0<t<t_{0}$,

$$
d_{\mathcal{P}}\left(\rho_{s, t}(x), \phi_{t}(x)\right)=d_{\mathcal{P}}\left(f_{s}(x), f_{s}\left(\phi_{t}(x)\right)\right)<\epsilon
$$

as required. 
We are now ready to prove Proposition 4.1 The idea of the proof is to combine two separate estimates, as we now sketch. On the one hand, we will use Lemma 4.4 to argue that we can make the integral over $X$ close to the analogous integral over $X_{0}$. On the other hand, we know from Lemma 4.3 that the integral over $X_{0}$ can be made arbitrarily small. Putting these estimates together gives the result. We make this precise in the proof below.

Proof of Proposition 4.1 We first note that by Lemma 4.3 there exists $s_{0}>0$ such that for any $s>s_{0}$ we have

$$
\int_{X_{0} \backslash B_{\eta, 0}}\left|\frac{\tilde{\chi}_{s}^{*}\left(\sigma^{\tilde{m}}\right)}{\left\|\tilde{\chi}_{s}^{*}\left(\sigma^{\tilde{m}}\right)\right\|_{L^{1}\left(X_{0}\right)}}\right| d(v o l)<\frac{\epsilon}{2} .
$$

Next we notice that since $\tilde{\chi}_{s}^{*}\left(\sigma^{\tilde{m}}\right)$ is a holomorphic section of a hermitian line bundle, its norm $\left|\tilde{\chi}_{s}^{*}\left(\sigma^{\tilde{m}}\right)\right|$ is a continuous function on $\mathcal{P}$. Since $\rho_{s, t}$ is continuous in $t$, as noted in Proposition 3.6 the function $t \mapsto$ $\left\|\tilde{\chi}_{s}^{*} \sigma^{\tilde{m}} \circ \rho_{s, t}\right\|_{L^{1}\left(X_{t}\right)}$ is a continous function on $t$. Define $C_{0}$ and $C_{1}$, respectively, to be the minumum and maximum values of $\left\|\tilde{\chi}_{s}^{*} \sigma^{\tilde{m}} \circ \rho_{s, t}\right\|_{L^{1}\left(X_{t}\right)}$ for $t \in[0,1]$, and note that $C_{0} \neq 0$ for sufficiently small $t$ because $\tilde{\chi}_{s}^{*}\left(\sigma^{\tilde{m}}\right)$ is not identically zero [7, Theorem $\left.5.3(\mathrm{a} 5)\right]$, and thus $\tilde{\chi}_{s}^{*} \sigma^{\tilde{m}} \circ \rho_{s, t}$ is not the zero section for sufficiently small $t$.

Similarly, since $\tilde{\chi}_{s}^{*}\left(\sigma^{\tilde{m}}\right)$ is a holomorphic section of a hermitian line bundle on the compact set $\mathcal{P}$, its norm $\left|\tilde{\chi}_{s}^{*}\left(\sigma^{\tilde{m}}\right)\right|$ is a continuous, and hence uniformly continuous, function on $\mathcal{P}$. Thus for any $\epsilon>0$, there exists a $\delta>0$ such that if $d_{\mathcal{P}}\left(x, x^{\prime}\right)<\delta$ for $x, x^{\prime} \in \mathcal{P}$, then

$$
|| \tilde{\chi}_{s}^{*}\left(\sigma^{\tilde{m}}\right)|(x)-| \tilde{\chi}_{s}^{*}\left(\sigma^{\tilde{m}}\right)\left|\left(x^{\prime}\right)\right|<\frac{\epsilon C_{0}\left\|\tilde{\chi}_{s}^{*} \sigma^{\tilde{m}}\right\|_{L^{1}\left(X_{0}\right)}}{4 C_{1} \operatorname{vol}\left(X_{t}\right)}
$$

Moreover, by Lemma 4.4, for a fixed $s$ with $s>s_{0}$ as above, we know there exists $t_{0}=t_{0}(s)>0$ such that for any $t$ with $0<t<t_{0}=t_{0}(s)$ and any $x \in X_{t}$ we have

$$
d_{\mathcal{P}}\left(\rho_{s, t}(x), \phi_{t}(x)\right)<\delta .
$$

For what follows we will also choose $t_{0}(s)$ sufficiently small so that $\tilde{\chi}_{s}^{*}\left(\sigma^{\tilde{m}}\right) \circ \rho_{s, t}$ is not identically zero for $0<t<t_{0}$, so in particular $C_{0}>0$. Now, we have that for all $x \in X_{t}$ with $0<t<t_{0}=t_{0}(s)$,

$$
|| \tilde{\chi}_{s}^{*}\left(\sigma^{\tilde{m}}\right)\left|\left(\rho_{s, t}(x)\right)-\right| \tilde{\chi}_{s}^{*}\left(\sigma^{\tilde{m}}\right)\left|\left(\phi_{t}(x)\right)\right|<\frac{\epsilon C_{0}\left\|\tilde{\chi}_{s}^{*} \sigma^{\tilde{m}}\right\|_{L^{1}\left(X_{0}\right)}}{4 C_{1} \operatorname{vol}\left(X_{t}\right)} \leq \frac{\epsilon\left\|\tilde{\chi}_{s}^{*} \sigma^{\tilde{m}}\right\|_{L^{1}\left(X_{0}\right)}}{4 \operatorname{vol}\left(X_{t}\right)}
$$

where the last inequality is because $C_{0} / C_{1} \leq 1$.

Next we recall that the sections $\sigma_{s, t}^{m}$ on $X$ in Definition 3.14 are given by a sequence of pullbacks. In particular, since both $\tilde{\phi}_{1-t}$ and $\tilde{\rho}_{s, t}$ preserve the hermitian metric and $\phi_{1-t}$ preserves symplectic structures, we have that

$$
\int_{X \backslash B_{\eta}}\left|\sigma_{s, t}^{m}\right| d(v o l)=\int_{X_{t} \backslash B_{\eta, t}}\left|\tilde{\rho}_{s, t}^{*} \tilde{\chi}_{s}^{*}\left(\sigma^{\tilde{m}}\right)\right| d(v o l)=\int_{X_{t} \backslash B_{\eta, t}}\left|\tilde{\chi}_{s}^{*}\left(\sigma^{\tilde{m}}\right)\right| \circ \rho_{s, t} d(v o l),
$$

where we also use that $\phi_{1-t}^{-1}\left(B_{\eta, t}\right)=B_{\eta}$ by construction (3.3) of the moment maps $\mu_{t}$; for the same reason, the $L^{1}$-norms satisfy

$$
\left\|\sigma_{s, t}^{m}\right\|_{L^{1}(X)}=\int_{X}\left|\sigma_{s, t}^{m}\right| d(v o l)=\int_{X_{t}}\left|\tilde{\chi}_{s}^{*}\left(\sigma^{\tilde{m}}\right)\right| \circ \rho_{s, t} d(v o l)=\left\|\tilde{\chi}_{s}^{*}\left(\sigma^{\tilde{m}}\right) \circ \rho_{s, t}\right\|_{L^{1}\left(X_{t}\right)} .
$$

Similarly, since $\phi_{t}$ and $\tilde{\phi}_{t}$ preserve the relevant structures we have

$$
\int_{X_{0} \backslash B_{\eta, 0}}\left|\tilde{\chi}_{s}^{*}\left(\sigma^{\tilde{m}}\right)\right| d(v o l)=\int_{X_{t} \backslash B_{\eta, t}}\left|\tilde{\phi}_{t}^{*} \tilde{\chi}_{s}^{*}\left(\sigma^{\tilde{m}}\right)\right| d(v o l)=\int_{X_{t} \backslash B_{\eta, t}}\left|\tilde{\chi}_{s}^{*}\left(\sigma^{\tilde{m}}\right)\right| \circ \phi_{t} d(v o l) .
$$


In this case, the $L^{1}$-norms satisfy

$$
\left\|\tilde{\chi}_{s}^{*}\left(\sigma^{\tilde{m}}\right)\right\|_{L^{1}\left(X_{0}\right)}=\int_{X_{0}}\left|\tilde{\chi}_{s}^{*}\left(\sigma^{\tilde{m}}\right)\right| d(v o l)=\int_{X_{t}}\left|\tilde{\chi}_{s}^{*}\left(\sigma^{\tilde{m}}\right)\right| \circ \phi_{t} d(v o l)=\left\|\tilde{\chi}_{s}^{*}\left(\sigma^{\tilde{m}}\right) \circ \phi_{t}\right\|_{L^{1}\left(X_{t}\right)} ;
$$

however, because $\phi_{t}$ preserves the symplectic structure, the above norms are equal for all $t \in[0,1]$.

Because of the normalizing factors in the denominators, we will need to show that $\left\|\tilde{\chi}_{s}^{*} \sigma^{\tilde{m}} \circ \rho_{s, t}\right\|_{L^{1}\left(X_{t}\right)}$ is close to $\left\|\tilde{\chi}_{s}^{*} \sigma^{\tilde{m}} \circ \phi_{t}\right\|_{L^{1}\left(X_{t}\right)}$, which we do as follows: For $s>s_{0}$ and for all $x$ in $X_{t}, 0<t \leq t_{0}(s)$,

$$
\begin{aligned}
\left|\left\|\tilde{\chi}_{s}^{*} \sigma^{\tilde{m}} \circ \rho_{s, t}\right\|-\left\|\tilde{\chi}_{s}^{*} \sigma^{\tilde{m}} \circ \phi_{t}\right\|\right| & \leq \int_{X_{t}}|| \tilde{\chi}_{s}^{*} \sigma^{\tilde{m}} \circ \rho_{s, t}|-| \tilde{\chi}_{s}^{*} \sigma^{\tilde{m}} \circ \phi_{t}|| d(v o l) \\
& \leq \int_{X_{t}} \frac{\epsilon C_{0}\left\|\tilde{\chi}_{s}^{*} \sigma^{\tilde{m}}\right\|_{L^{1}\left(X_{0}\right)}}{4 C_{1} \operatorname{vol}\left(X_{t}\right)} d(\mathrm{vol})=\frac{\epsilon C_{0}\left\|\tilde{\chi}_{s}^{*} \sigma^{\tilde{m}}\right\|_{L^{1}\left(X_{0}\right)}}{4 C_{1}}
\end{aligned}
$$

where the last inequality comes from (4.4).

Now, using the fact that

$$
\left|\frac{a}{h}-\frac{b}{k}\right|=\left|\frac{a(k-h)+h(a-b)}{h k}\right| \leq \frac{|a||k-h|}{|h k|}+\frac{|a-b|}{|k|}
$$

we obtain that, for our fixed value of $s>s_{0}$ and for all $x \in X_{t}$ with $0<t \leq t_{0}(s)$, the expression

$$
\left|\frac{\left|\tilde{\chi}_{s}^{*} \sigma^{\tilde{m}}\right|\left(\rho_{s, t}(x)\right)}{\left\|\tilde{\chi}_{s}^{*} \sigma^{\tilde{m}} \circ \rho_{s, t}\right\|}-\frac{\left|\tilde{\chi}_{s}^{*} \sigma^{\tilde{m}}\right|\left(\phi_{t}(x)\right)}{\left\|\tilde{\chi}_{s}^{*} \sigma^{\tilde{m}} \circ \phi_{t}\right\|}\right|
$$

is less than or equal to

$$
\frac{\left|\tilde{\chi}_{s}^{*} \sigma^{\tilde{m}}\left(\rho_{s, t}(x)\right)\right|}{\left\|\tilde{\chi}_{s}^{*} \sigma^{\tilde{m}} \circ \rho_{s, t}\right\|\left\|\tilde{\chi}_{s}^{*} \sigma^{\tilde{m}} \circ \phi_{t}\right\|}\left|\left\|\tilde{\chi}_{s}^{*} \sigma^{\tilde{m}} \circ \rho_{s, t}\right\|-\left\|\tilde{\chi}_{s}^{*} \sigma^{\tilde{m}} \circ \phi_{t}\right\|\right|+\frac{|| \tilde{\chi}_{s}^{*}\left(\sigma^{\tilde{m}}\right)\left|\left(\rho_{s, t}(x)\right)-\right| \tilde{\chi}_{s}^{*}\left(\sigma^{\tilde{m}}\right)\left|\left(\phi_{t}(x)\right)\right|}{\left\|\tilde{\chi}_{s}^{*} \sigma^{\tilde{m}} \circ \phi_{t}\right\|} .
$$

Using (4.9) on the first term, the second estimate in (4.4) on the second term, the fact that $\left|\tilde{\chi}_{s}^{*}\left(\sigma^{\tilde{m}}\right)\left(\rho_{s, t}(x)\right)\right| \leq$ $\frac{C_{1}}{\operatorname{vol}\left(X_{t}\right)}$ by the definition of $C_{1}$, the fact that $C_{0}$ is the minimum value of $\left\|\tilde{\chi}_{s}^{*} \sigma^{\tilde{m}} \circ \rho_{s, t}\right\|$ (and hence $\frac{1}{\left\|\tilde{\chi}_{s}^{*} \sigma^{\tilde{m}} \circ \rho_{s, t}\right\|} \leq$ $\left.\frac{1}{C_{0}}\right)$, and the equality (4.8), this becomes

$$
\begin{aligned}
\left|\frac{\left|\tilde{\chi}_{s}^{*} \sigma^{\tilde{m}}\right|\left(\rho_{s, t}(x)\right)}{\left\|\tilde{\chi}_{s}^{*} \sigma^{\tilde{m}} \circ \rho_{s, t}\right\|}-\frac{\left|\tilde{\chi}_{s}^{*} \sigma^{\tilde{m}}\right|\left(\phi_{t}(x)\right)}{\left\|\tilde{\chi}_{s}^{*} \sigma^{\tilde{m}} \circ \phi_{t}\right\|}\right| & \leq \frac{C_{1}}{C_{0} \operatorname{vol}\left(X_{t}\right)\left\|\tilde{\chi}_{s}^{*} \sigma^{\tilde{m}} \circ \phi_{t}\right\|} \frac{\epsilon C_{0}\left\|\tilde{\chi}_{s}^{*} \sigma^{\tilde{m}}\right\|}{4 C_{1}}+\frac{1}{\left\|\tilde{\chi}_{s}^{*} \sigma^{\tilde{m}} \circ \phi_{t}\right\|} \frac{\epsilon\left\|\tilde{\chi}_{s}^{*} \sigma^{\tilde{m}}\right\|}{4 \operatorname{vol}\left(X_{t}\right)} \\
& =\frac{\epsilon}{2 \operatorname{vol}\left(X_{t}\right)}
\end{aligned}
$$


Putting everything together, for $s>s_{0}$ and $t<t_{0}$ for the chosen $s_{0}, t_{0}(s)$ as above, we have

$$
\begin{aligned}
& \int_{X \backslash B_{\eta}} \frac{\left|\sigma_{s, t}^{m}\right|}{\left\|\sigma_{s, t}^{m}\right\|} d(\mathrm{vol})=\int_{X \backslash B_{\eta}} \frac{\left|\sigma_{s, t}^{m}\right|}{\left\|\sigma_{s, t}^{m}\right\|} d(\mathrm{vol})-\int_{X_{0} \backslash B_{\eta, 0}} \frac{\left|\tilde{\chi}_{s}^{*}\left(\sigma^{\tilde{m}}\right)\right|}{\left\|\tilde{\chi}_{s}^{*}\left(\sigma^{\tilde{m}}\right)\right\|} d(\mathrm{vol})+\int_{X_{0} \backslash B_{\eta, 0}} \frac{\left|\tilde{\chi}_{s}^{*}\left(\sigma^{\tilde{m}}\right)\right|}{\left\|\tilde{\chi}_{s}^{*}\left(\sigma^{\tilde{m}}\right)\right\|} d(\mathrm{vol}) \\
& =\int_{X_{t} \backslash B_{\eta, t}} \frac{\left|\tilde{\chi}_{s}^{*}\left(\sigma^{\tilde{m}}\right)\right| \circ \rho_{s, t}}{\left\|\tilde{\chi}_{s}^{*}\left(\sigma^{\tilde{m}}\right) \circ \rho_{s, t}\right\|} d(\mathrm{vol})-\int_{X_{t} \backslash B_{\eta, t}} \frac{\left|\tilde{\chi}_{s}^{*}\left(\sigma^{\tilde{m}}\right)\right| \circ \phi_{t}}{\left\|\tilde{\chi}_{s}^{*}\left(\sigma^{\tilde{m}}\right) \circ \phi_{t}\right\|} d(\mathrm{vol})+\int_{X_{0} \backslash B_{\eta, 0}} \frac{\left|\tilde{\chi}_{s}^{*}\left(\sigma^{\tilde{m}}\right)\right|}{\left\|\tilde{\chi}_{s}^{*}\left(\sigma^{\tilde{m}}\right)\right\|} d(\mathrm{vol}) \\
& \leq\left|\int_{X_{t} \backslash B_{\eta, t}} \frac{\left|\tilde{\chi}_{s}^{*}\left(\sigma^{\tilde{m}}\right)\right| \circ \rho_{s, t}}{\left\|\tilde{\chi}_{s}^{*}\left(\sigma^{\tilde{m}}\right) \circ \rho_{s, t}\right\|} d(\mathrm{vol})-\int_{X_{t} \backslash B_{\eta, t}} \frac{\left|\tilde{\chi}_{s}^{*}\left(\sigma^{\tilde{m}}\right)\right| \circ \phi_{t}}{\left\|\tilde{\chi}_{s}^{*}\left(\sigma^{\tilde{m}}\right) \circ \phi_{t}\right\|} d(\mathrm{vol})\right|+\int_{X_{0} \backslash B_{\eta, 0}} \frac{\left|\tilde{\chi}_{s}^{*}\left(\sigma^{\tilde{m}}\right)\right|}{\left\|\tilde{\chi}_{s}^{*}\left(\sigma^{\tilde{m}}\right)\right\|} d(\mathrm{vol}) \\
& \leq \int_{X_{t} \backslash B_{\eta, t}}\left|\frac{\left|\tilde{\chi}_{s}^{*}\left(\sigma^{\tilde{m}}\right)\right| \circ \rho_{s, t}}{\left\|\tilde{\chi}_{s}^{*}\left(\sigma^{\tilde{m}}\right) \circ \rho_{s, t}\right\|}-\frac{\left|\tilde{\chi}_{s}^{*}\left(\sigma^{\tilde{m}}\right)\right| \circ \phi_{t}}{\left\|\tilde{\chi}_{s}^{*}\left(\sigma^{\tilde{m}}\right) \circ \phi_{t}\right\|}\right| d(v o l)+\int_{X_{0} \backslash B_{\eta, 0}} \frac{\left|\tilde{\chi}_{s}^{*}\left(\sigma^{\tilde{m}}\right)\right|}{\left\|\tilde{\chi}_{s}^{*}\left(\sigma^{\tilde{m}}\right)\right\|} d(\mathrm{vol}) \\
& \leq \int_{X_{t} \backslash B_{\eta, t}} \frac{\epsilon}{2 \operatorname{vol}\left(X_{t}\right)} d(v o l)+\int_{X_{0} \backslash B_{\eta, 0}} \frac{\left|\tilde{\chi}_{s}^{*}\left(\sigma^{\tilde{m}}\right)\right|}{\left\|\tilde{\chi}_{s}^{*}\left(\sigma^{\tilde{m}}\right)\right\|} d(v o l) \\
& \leq \frac{\epsilon}{2 \operatorname{vol}\left(X_{t}\right)} \cdot \operatorname{vol}\left(X_{t}\right)+\frac{\epsilon}{2}=\epsilon
\end{aligned}
$$

as required, where the second equality uses (4.5), (4.6), (4.7), and (4.8), the second-to-last inequality uses (4.11), and the last inequality uses (4.3).

Finally, we wish to prove that there exists $t^{\prime}=t^{\prime}(s):[0, \infty) \rightarrow[0,1]$ a continuous function of $s$ such that (4.12) holds for $\sigma_{s, t}^{m}$ for all $s$ and all $t$ with $0 \leq t \leq t^{\prime}(s)$, and also such that $t^{\prime}(s) \rightarrow 0$ as $s \rightarrow \infty$. To see this, notice first that immediately before (4.4) we made a choice of $t_{0}(s)$ which depended on $s$. In the subsequent argument we proved statements that hold for all $t$ with $0<t<t_{0}$. In particular, these statements are still true if we replace $t_{0}$ by a smaller choice of $t_{0}$. From this it follows that we may assume without loss of generality that $t_{0}=t_{0}(s)$ is a monotone non-increasing function of $s$.

A standard result from real analysis (see e.g. [21, Lemma 1.6.31(iii)]) says that a bounded, non-decreasing monotone function $F: \mathbb{R} \rightarrow \mathbb{R}$ can be written as $F=F_{c}+F_{p p}$, where $F_{c}$ is a continuous monotone nondecreasing function and $F_{p p}$ is a jump function. Looking at the definition of $F_{p p}$ in the proof in [21], it is obvious that $F_{p p}$ is nonnegative; a little more thought shows that if $F(x)$ is nonnegative then $F_{p p}(x) \leq F(x)$ for all $x$, so if $F(x)$ is nonnegative then so is $F_{c}$. Taking $F(s)=t_{0}(-s)$ for $s \leq 0$ (and constant for $s \geq 0$ ), we can apply this decomposition to obtain that $t_{0}(s)$ for $s>0$ is the sum of a nonnegative jump function and a continuous positive non-increasing function $t^{\prime}(s)$ which therefore satisfies $0 \leq t^{\prime}(s) \leq t_{0}(s)$. By shrinking $t^{\prime}(s)$ if necessary, we can arrange that $t^{\prime}(s) \rightarrow 0$ as $s \rightarrow \infty$. Since the inequalities at each stage are true for the chosen $s$ and for all $t$ with $0<t \leq t_{0}(s)$, they are true for $0<t<t^{\prime}(s)$, and we are finished.

Next, the fiber $\mu_{0}^{-1}(m)$ is diffeomorphic to a torus and lies entirely within the open dense torus orbit of $X_{0}$, and thus it is possible to obtain much more refined information about the behavior of the family $\left\{\sigma_{s, t}^{m}\right\}$ in the limit. Specifically, let $\Gamma\left(X, L^{*}\right)$ denote the space of smooth (not necessarily holomorphic) sections of the dual complex line bundle and let $\langle\cdot, \cdot\rangle$ denote the usual pairing between $L^{*}$ and $L$. For $\sigma \in \Gamma\left(X, L^{*}\right)$ we let $\|\sigma\|_{L^{1}(X)}$ denote its $L^{1}$-norm with respect to the Hermitian metric on $L$, i.e. $\|\sigma\|_{L^{1}(X)}=\int_{X}|\sigma| d(v o l)$. We have the following.

Proposition 4.5. Let $m \in \Delta_{0} \cap \mathbb{Z}^{n}$ be an interior lattice point. Let $\tau \in \Gamma\left(X_{1}, L_{1}^{*}\right)$. Then there exist a covariantly constant section $\delta_{m}$ of $\left(\left.L\right|_{\mu^{-1}(m)},\left.\nabla\right|_{\mu^{-1}(m)}\right)$, a measure $d \theta_{m}$ on $\mu^{-1}(m)$, and a continuous function $t=t(s)$ satisfying $\lim _{s \rightarrow \infty} t(s)=0$ such that

$$
\lim _{s \rightarrow \infty} \int_{X}\left\langle\tau, \frac{\sigma_{s, t(s)}^{m}}{\left\|\sigma_{s, t(s)}^{m}\right\|_{L^{1}(X)}}\right\rangle d(v o l)=\int_{\mu^{-1}(m)}\left\langle\tau, \delta_{m}\right\rangle d \theta_{m} .
$$


The idea of the proof is similar to that of Proposition 4.1 above and requires a number of steps. Namely, we will relate the LHS of (4.13) to a limit of integrals on $X_{t(s)}$ and then approximate the integral on $X_{t(s)}$ by one on $X_{0}$. We then use the fact that the analogous statement to Proposition 4.5 is already known on $X_{0}$; this is the content of the following lemma.

Lemma 4.6. Let $U_{0}$ denote the open dense $\mathbb{T}_{0}$-orbit in $X_{0}$ and let $m \in \Delta_{0} \cap \mathbb{Z}^{n}$ be an interior lattice point. Let $\tilde{m}$ be the fixed choice of preimage of $m$ under $\iota^{*}$ as in Definition 3.14 Then there exists a covariantly constant section $\delta_{m, 0}$ of $\left(\left.L_{\mathcal{P}}\right|_{\mu_{0}^{-1}(m)},\left.\nabla\right|_{\mu_{0}^{-1}(m)}\right)$ over $\mu_{0}^{-1}(m)$ and a measure $d \theta_{m, 0}$ on $\mu_{0}^{-1}(m)$ such that, for any smooth section $\tau \in \Gamma\left(U_{0},\left.L_{\mathcal{P}}^{*}\right|_{V_{0}}\right)$ of the dual line bundle, we have

$$
\lim _{s \rightarrow \infty} \int_{X_{0}}\left\langle\tau, \frac{\tilde{\chi}_{s}^{*}\left(\sigma^{\tilde{m}}\right)}{\left\|\tilde{\chi}_{s}^{*}\left(\sigma^{\tilde{m}}\right)\right\|_{L^{1}\left(X_{0}\right)}}\right\rangle d(\mathrm{vol})=\int_{\mu_{0}^{-1}(m)}\left\langle\tau, \delta_{m, 0}\right\rangle d \theta_{m, 0} .
$$

Proof. This is essentially the content of an argument given in [7]. Specifically, the $V_{\text {symp }}$ and $V_{\text {comp }}$ in the proof of [7. Proposition 6.6(4)] can be identified with our $X_{0}$. Similarly, their $T_{\mathbb{C}}^{\ell}$ (respectively $T_{\mathbb{C}}^{n}$ ) is our $\mathbb{T}_{0}$ (respectively $\mathbb{T}_{\mathcal{P}}$ ). Finally, to apply the argument in $[7]$ it is necessary that $X_{0}$ is the closure of the $\mathbb{T}_{0}$-orbit of $[1: 1: \cdots: 1]$ and that $\iota^{*}$ is surjective on lattices, which hold by our assumptions (e) and (g), respectively, as stated in Section 2] Thus, the argument of [7] applies.

In order to translate the previous lemma to a statement concerning other fibers, we need some additional information. The next lemma recalls some results from [8] and also constructs compact subsets $K_{t}$ which will be useful for obtaining estimates. Let $\Delta_{0}^{\circ}$ denote the interior of the moment polytope.

Lemma 4.7. Let $t \in[0,1]$. Then there exists an open subset $U_{t} \subseteq X_{t}$ and a compact subset $K_{t} \subseteq U_{t}$ such that:

(1) For $t=0, U_{0}$ equals $\mu_{0}^{-1}\left(\Delta_{0}^{\circ}\right) \subseteq X_{0}$, the open dense $\mathbb{T}_{0}$-orbit in $X_{0}$. In particular, $U_{0}$ lies in the smooth locus of $X_{0}$.

(2) The gradient-Hamiltonian flow $\phi_{s}: U_{t} \rightarrow U_{t-s}$ is a diffeomorphism for all $0 \leq s \leq t$. In particular, $\phi_{t}$ is a diffeomorphism from $U_{t}$ to $U_{0}$.

(3) The flow $\phi_{s}: U_{t} \rightarrow U_{t-s}$ in (2) identifies $K_{t}$ with $K_{t-s}$, i.e., $\phi_{s}\left(K_{t}\right)=K_{t-s}$ for all $0 \leq s \leq t$.

(4) The subset $K_{t}$ contains a neighborhood of every interior Bohr-Sommerfeld fiber. More precisely, there exists some $\eta>0$ such that, for any $m \in \Delta_{0}^{\circ} \cap \mathbb{Z}^{n}$, the neighborhood $B_{\eta, t}(m)$ as in (4.1) is contained in $K_{t}$.

Proof. In order to satisfy (1), we first define $U_{0}:=\mu_{0}^{-1}\left(\Delta_{0}^{\circ}\right)$. From [8, Corollary 3.3] it follows that $U_{0}$ is contained in the locus of points in $X$ where the gradient-Hamiltonian vector field is defined. Moreover, as in the proof of [8, Theorem 5.2], we know the gradient-Hamiltonian flow is well-defined on all of $X_{t}$ for any $t \neq 0$. By [8, Lemma 2.5] we may now define $U_{t}:=\phi_{-t}\left(U_{0}\right)=\phi_{t}^{-1}\left(U_{0}\right)$ from which it is immediate that $\phi_{s}: U_{t} \rightarrow U_{t-s}$ is a diffeomorphism from $U_{t}$ to $U_{t-s}$ (with inverse $\phi_{-s}$ ) for any $0 \leq s \leq t$. This proves (2).

It remains to define the compact subsets $K_{t}$ and to prove the claims (3) and (4). Let $C \subset \Delta_{0}^{\circ}$ be a connected closed subset containing within its interior every interior lattice point, i.e., if $m \in \Delta_{0}^{\circ} \cap \mathbb{Z}^{n}$ then $m \in C^{\circ}$; such a $C$ clearly exists. We define $K_{t}:=\mu_{t}^{-1}(C)$. Then $K_{t}$ is closed since $\mu_{t}$ is continuous. Since $C \subseteq \Delta_{0}^{\circ}$ and we saw $\mu_{0}^{-1}\left(\Delta_{0}^{\circ}\right) \subseteq U_{0}$ above, it also follows from the definition (3.3) of the $\mu_{t}$ that $K_{t} \subseteq U_{t}$. Moreover, since $\mu_{t-s} \circ \phi_{s}=\mu_{t}$ by construction of the integrable systems (3.3), and the $\phi_{t}$ are diffeomorphisms on the $U_{t}$, it follows that $\phi_{s}\left(K_{t}\right)=K_{t-s}$ for all $0 \leq s \leq t$. This proves (3). Finally, since $C$ contains only a finite number of lattice points, there exists some $\eta>0$ such that for all interior lattice points $m$, the ball $B_{\eta}(m)$ is contained in $C$. This proves (4) and completes the proof.

Roughly, the idea in what follows is to replace the integrals in previous proofs by integrals over $K_{t}$. By using Proposition 4.1 we will be able to control the error terms. Then, since $K_{t}$ is compact by assumption, we will be able to use a uniform continuity argument. We begin with an estimate which is uniform on $K_{t}$ for all sufficiently small $t$. This will be a key component of the proof of Proposition 4.5. 
Lemma 4.8. Let $\tilde{m}$ be a preimage of an interior lattice point $m \in \Delta_{0} \cap \mathbb{Z}^{n}$. There exists a continuous function $t^{\prime \prime}=t^{\prime \prime}(s): \mathbb{R}_{>0} \rightarrow[0,1]$ such that for any $\varepsilon>0$ and any $s \in \mathbb{R}_{>0}$, the following holds: if $t \in[0,1]$ satisfies $0 \leq t \leq t^{\prime \prime}(s)$ then

$$
\left|\frac{\tilde{\rho}_{s, t}^{*} \tilde{\chi}_{s}^{*}\left(\sigma^{\tilde{m}}\right)(x)}{\left\|\tilde{\rho}_{s, t}^{*} \tilde{\chi}_{s}^{*}\left(\sigma^{\tilde{m}}\right)\right\|_{L^{1}\left(X_{t}\right)}}-\frac{\tilde{\phi}_{t}^{*} \tilde{\chi}_{s}^{*}\left(\sigma^{\tilde{m}}\right)(x)}{\left\|\tilde{\phi}_{t}^{*} \tilde{\chi}_{s}^{*}\left(\sigma^{\tilde{m}}\right)\right\|_{L^{1}\left(X_{t}\right)}}\right|<\varepsilon
$$

for all $x \in K_{t}$.

Proof. The idea of the proof is to show that the LHS of (4.15) is (the norm of) a continuous section of a line bundle over a suitable family that is equal to zero for all $x \in K_{0}$ (i.e. when $t=0$ ) for any value of $s$; then we use uniform continuity.

Recall that $X_{[0,1]}$ denotes the restriction of our family $X_{\text {to }}$ the subset $[0,1] \subseteq \mathbb{C}$, i.e. $X_{[0,1]}:=\pi^{-1}([0,1]) \subset$ $X$, and $\mathcal{U}_{[0,1]}=\left\{x \in \mathcal{X}_{[0,1]} \mid x \in U_{\pi(x)}\right\}$ is the family of open dense subsets $U_{t}$ in each fibre, as in (3.4). We also define

$$
\mathcal{K}_{[0,1]}:=\left\{x \in \mathcal{X}_{[0,1]} \mid x \in K_{\pi(x)}\right\}
$$

to be the family of the compact sets $K_{t}$ from Lemma4.7 over [0,1]. Let $L_{\mathcal{K}}$ (respectively $L_{\varkappa}$ ) denote $\left.L_{X}\right|_{\mathcal{K}_{[0,1]}}$ (respectively $L_{X} \mid u_{[0,1]}$ ).

Now fix $s \in \mathbb{R}$ with $s>0$ and let $x \in U_{t} \subset X_{t}$ for any $t$. Recall that $\phi_{t}$ is the gradient-Hamiltonian flow, so in particular $\phi_{t}: X_{t} \rightarrow X_{0}$ takes $X_{t}$ to $X_{0}$ and $U_{t}$ to $U_{0}$ (cf. Lemma 4.7). In the LHS of (4.15), the expression $\tilde{\phi}_{t}^{*} \tilde{\chi}_{s}^{*}\left(\sigma^{\tilde{m}}\right)(x)$ is by definition equal to $\left(\left.\tilde{\phi}_{t}^{-1} \circ \tilde{\chi}_{s}^{*}\left(\sigma^{\tilde{m}}\right)\right|_{X_{0}} \circ \phi_{t}\right)(x)$. In particular, $\tilde{\phi}_{t}^{*} \tilde{\chi}_{s}^{*}\left(\sigma^{\tilde{m}}\right)$ sends each $U_{t}$ to $L_{t}$ by definition, so it is a section of the bundle $L_{t} \rightarrow U_{t}$. Putting these together for all $U_{t}$ for $t \in[0,1]$, we obtain a section of $L_{\mathfrak{U}} \rightarrow \mathcal{U}_{[0,1]}$. The $L^{1}$-norm in the denominator is independent of $t$, since it can be written as

$$
\left\|\tilde{\phi}_{t}^{*} \tilde{\chi}_{s}^{*}\left(\sigma^{\tilde{m}}\right)\right\|_{L^{1}\left(X_{t}\right)}=\int_{X_{t}}\left|\tilde{\phi}_{t}^{*} \tilde{\chi}_{s}^{*}\left(\sigma^{\tilde{m}}\right)\right| d(v o l)=\int_{X_{0}}\left|\tilde{\chi}_{s}^{*}\left(\sigma^{\tilde{m}}\right)\right| d(v o l)
$$

since $\phi_{t}$ preserves the Hermitian and symplectic structures, and so the denominator of the second term is constant on $\mathcal{U}_{[0,1]}$.

Recall that $\sigma^{m}$ is holomorphic (hence smooth), $\chi_{s}$ is smooth, and the map $\phi: \mathcal{U}_{[0,1]} \rightarrow U_{0}$ given by $x \mapsto \phi_{\pi(x)}(x)$ is smooth (since it is the flow of a smooth vector field). Together with the last statement in Lemma 3.7 we may conclude that the section of $L_{\mathcal{U}} \rightarrow \mathcal{U}_{[0,1]}$ obtained above (sending $x \in U_{t}$ to $\left.\tilde{\phi}_{\pi(x)}^{*} \tilde{\chi}_{s}^{*}\left(\sigma^{\tilde{m}}\right)(x)\right)$ is smooth. Finally, recalling that when $t=0$ the maps $\phi_{0}: U_{0} \rightarrow U_{0}$ and $\tilde{\phi}_{0}:\left.\left.L_{0}\right|_{U_{0}} \rightarrow L_{0}\right|_{U_{0}}$ are both equal to the identity, we conclude that the above section restricts on $U_{0}$ to be equal to $\left.\frac{\tilde{\chi}_{s}^{*}\left(\sigma^{\tilde{m}}\right)}{\left\|\tilde{\chi}_{s}^{*}\left(\sigma^{m}\right)\right\|}\right|_{U_{0}}$.

Next, we consider the expression $\tilde{\rho}_{s, t}^{*} \tilde{\chi}_{s}^{*}\left(\sigma^{\tilde{m}}\right)$ contained in the LHS of (4.15). Recall that $\rho_{s, t}$ is the embedding $X_{t} \rightarrow \mathcal{P}$ given in (3.6) and specified in Proposition 3.2. and $\tilde{\rho}_{s, t}$ is the lift of $\rho_{s, t}$ to the line bundles (cf. Lemma 3.8). In particular, for $x \in U_{t}$ the expression $\tilde{\rho}_{s, t}^{*} \tilde{\chi}_{s}^{*}\left(\sigma^{\tilde{m}}\right)(x)$ is by definition equal to $\left(\left.\tilde{\rho}_{s, t}^{-1} \circ \tilde{\chi}_{s}\left(\sigma^{\tilde{m}}\right)\right|_{\rho_{s, t}\left(U_{t}\right)} \circ \rho_{s, t}\right)(x)$. As in the above case, by construction $\tilde{\rho}_{s, t}^{*} \circ \tilde{\chi}_{s}^{*}\left(\sigma^{\tilde{m}}\right)$ sends each $U_{t}$ to $L_{t}$ by definition, so it is a section of $L_{t} \rightarrow U_{t}$ and by putting these together we obtain a section of $L_{\mathfrak{U}} \rightarrow \mathcal{U}_{[0,1]}$. Similarly to the above case, the last statement of Proposition 3.9 allows us to conclude that this section is continuous. In this case the $L^{1}$-norm in the denominator is no longer independent of $t$, but it will be continuous in $t$, and never zero since the section is not the zero section. Moreover, by our assumption on $\left\{\chi_{s}\right\}$ and Proposition 3.5 as well as Proposition 3.9 we also know that $\rho_{s, 0}=\mathrm{id}$ for all $s$ and its lift $\tilde{\rho}_{s, 0}$ acts as the identity on $\left.L_{0}\right|_{U_{0}}$, so $\tilde{\rho}_{s, 0}^{*} \tilde{\chi}_{s}^{*}\left(\sigma^{\tilde{m}}\right)=\tilde{\chi}_{s}^{*}\left(\sigma^{\tilde{m}}\right)$.

Now for $x \in \mathcal{K}_{[0,1]}$, let

$$
h_{s}(x)=\frac{\tilde{\rho}_{s, t}^{*} \tilde{\chi}_{s}^{*}\left(\sigma^{\tilde{m}}\right)(x)}{\left\|\tilde{\rho}_{s, t}^{*} \tilde{\chi}_{s}^{*}\left(\sigma^{\tilde{m}}\right)\right\|}-\frac{\tilde{\phi}_{t}^{*} \tilde{\chi}_{s}^{*}\left(\sigma^{\tilde{m}}\right)(x)}{\left\|\tilde{\phi}_{t}^{*} \tilde{\chi}_{s}^{*}\left(\sigma^{\tilde{m}}\right)\right\|} .
$$


By the preceding discussion, $h_{s}$ is a continuous section of $L_{\mathcal{K}}$ over $\mathcal{K}_{[0,1]}$ and in particular its absolute value $\left|h_{s}\right|$ is a continuous function from $\mathcal{K}_{[0,1]}$ to $\mathbb{R}$. Moreover, by the above, $h_{s}(x)=0$ for $x \in X_{0}$.

By the continuity of $h_{s}$, and since we know $K_{0} \subseteq h_{s}^{-1}(0)$, we may cover $K_{0}$ with open sets $U_{\alpha}$ with the property that $\left|h_{s}(x)\right|<\varepsilon$ for all $U_{\alpha}$. Extend this to an open cover of $\mathcal{K}_{[0,1]}$. The compactness of $\mathcal{K}_{[0,1]}$ implies that there exists a finite subcover, a subset of which is a finite cover of the subset $K_{0}$ of $\mathcal{K}_{[0,1]}$. In particular, from the construction of the cover, we may conclude that there exists some $t^{\prime \prime}(s)>0$ such that for all $0 \leq t<t^{\prime \prime}(s)$ and $x \in K_{t}$ we have $\left|h_{s}(x)\right|<\varepsilon$. Now an argument similar to that in the proof of Proposition 4.1 shows that in fact we may choose $t^{\prime \prime}(s)$ to be a continuous function of $s$, as claimed.

We are finally ready for a proof of Proposition 4.5 .

Proof of Proposition 4.5 We first define the covariantly constant section $\delta_{m}$ and measure $d \theta_{m}$. Recall that the integrable system on the variety $X$ is defined by pulling back that on $X_{0}$ via $\phi_{1}$. In particular $\phi_{1}$ induces a pullback of the action-angle coordinates on $U_{0}$ to $U=U_{1}=\phi_{1}^{-1}\left(U_{0}\right)$ and a diffeomorphism of tori $\mu_{0}^{-1}(m) \cong \mu^{-1}(m) \cong\left(S^{1}\right)^{n}$. Hence by using $\phi_{1}$ we may pull back the covariantly constant $\delta_{m, 0}$ and measure $d \theta_{m, 0}$ on $U_{0}$ of Lemma 4.6 to a covariantly constant $\delta_{m}$ and $d \theta_{m}$ respectively. Let $\tau \in \Gamma\left(U,\left.L_{\mathcal{P}}^{*}\right|_{U}\right)$ be a test section. For simplicity of notation we let $\left(\phi_{1}\right)_{*} \tau$ denote the section of $\left.L_{\mathcal{P}}^{*}\right|_{U_{0}}$ obtained by using the identifications $\phi_{1}: U=U_{1} \rightarrow U_{0}$ and $\tilde{\phi}_{1}:\left.\left.L_{\mathcal{P}}\right|_{U} \rightarrow L_{\mathcal{P}}\right|_{U_{0}}$, so $\left(\phi_{1}\right)_{*} \tau(x):=\tilde{\phi}_{1} \circ \tau \circ \phi_{1}^{-1}(x)$. Then by definition we have

$$
\int_{\mu_{0}^{-1}(m)}\left\langle\left(\phi_{1}\right)_{*} \tau, \delta_{m, 0}\right\rangle d \theta_{m, 0}=\int_{\mu^{-1}(m)}\left\langle\tau, \delta_{m}\right\rangle d \theta_{m}
$$

for any test section. Now let $\epsilon>0$. From the above it suffices to prove that we can find a continuous function $t=t(s)$ and an $s_{0}>0$ such that for all $s>s_{0}$ we have

$$
\left|\int_{X}\left\langle\tau, \frac{\sigma_{s, t(s)}^{m}}{\left\|\sigma_{s, t(s)}^{m}\right\|}\right\rangle d(v o l)-\int_{\mu_{0}^{-1}(m)}\left\langle\left(\phi_{1}\right)_{*} \tau, \delta_{m, 0}\right\rangle d \theta_{m, 0}\right|<\epsilon .
$$

First, we know from Lemma 4.6 that there exists $s_{1}$ such that for all $s>s_{1}$ we have

$$
\left|\int_{X_{0}}\left\langle\left(\phi_{1}\right)_{*} \tau, \frac{\tilde{\chi}_{s}^{*}\left(\sigma^{\tilde{m}}\right)}{\left\|\tilde{\chi}_{s}^{*}\left(\sigma^{\tilde{m}}\right)\right\|}\right\rangle d(v o l)-\int_{\mu_{0}^{-1}(m)}\left\langle\left(\phi_{1}\right)_{*} \tau, \delta_{m, 0}\right\rangle d \theta_{m, 0}\right|<\frac{\epsilon}{4} .
$$

Moreover, since $\phi_{1}=\phi_{t} \circ \phi_{1-t}$ and all maps preserve the relevant structures, we have

$$
\left|\int_{X_{t}}\left\langle\left(\phi_{1-t}\right)_{*} \tau, \frac{\tilde{\phi}_{t}^{*} \tilde{\chi}_{s}^{*}\left(\sigma^{\tilde{m}}\right)}{\left\|\tilde{\phi}_{t}^{*} \tilde{\chi}_{s}^{*}\left(\sigma^{\tilde{m}}\right)\right\|}\right\rangle d(v o l)-\int_{\mu_{0}^{-1}(m)}\left\langle\left(\phi_{1}\right)_{*} \tau, \delta_{m, 0}\right\rangle d \theta_{m, 0}\right|<\frac{\epsilon}{4} .
$$

Next note that $\tau$ is a smooth section on the compact space $X$, so there exists a constant $C>0$ such that $\|\tau\|<C$ for all $x \in X$. Since $\phi_{1-t}$ preserves the Hermitian structure, this also implies that $\left|\left(\tilde{\phi}_{1-t}\right)_{*} \tau\right|<C$ for all $x \in X_{t}$ and for all $t$. Let $K=K_{1}$ be the compact subset of $X=X_{1}$ from Lemma 4.7. Our next step is to approximate the integrals over $X$ and $X_{0}$ with integrals over $K$ and $K_{0}$. Specifically, following Lemma 4.7 (4) let $\eta>0$ be such that such that $B_{\eta}=B_{\eta}(m) \subset K$. By Proposition 4.1, there exists $s_{2}>0$ such that

$$
\int_{X \backslash B_{\eta}}\left|\frac{\sigma_{s, t}^{m}}{\left\|\sigma_{s, t}^{m}\right\|}\right| d(v o l)<\frac{\epsilon}{4 C}
$$

for any $s>s_{2}$ and $0 \leq t \leq t^{\prime}(s)$ where $t=t^{\prime}(s)$ is the function constructed in the proof of Proposition 4.1. Since $B_{\eta} \subset K$ and because we have an upper bound $|\tau|<C$ on the norm of $\tau$, we conclude

$$
\int_{X \backslash K}\left|\frac{\sigma_{s, t}^{m}}{\left\|\sigma_{s, t}^{m}\right\|}\right||\tau|<\int_{X \backslash B_{\eta}}\left|\frac{\sigma_{s, t}^{m}}{\left\|\sigma_{s, t}^{m}\right\|}\right||\tau|<\frac{\epsilon}{4}
$$


for $s>s_{2}$ and $0 \leq t \leq t^{\prime}(s)$. Thus

$$
\begin{aligned}
\left|\int_{X}\left\langle\tau, \frac{\sigma_{s, t}^{m}}{\left\|\sigma_{s, t}^{m}\right\|}\right\rangle d(\mathrm{vol})-\int_{K}\left\langle\tau, \frac{\sigma_{s, t}^{m}}{\left\|\sigma_{s, t}^{m}\right\|}\right\rangle d(\mathrm{vol})\right| & =\left|\int_{X \backslash K}\left\langle\tau, \frac{\sigma_{s, t}^{m}}{\left\|\sigma_{s, t}^{m}\right\|}\right\rangle d(\mathrm{vol})\right| \\
& \leq \int_{X \backslash K}|\tau|\left|\frac{\sigma_{s, t}^{m}}{\left\|\sigma_{s, t}^{m}\right\|}\right| d(\mathrm{vol})<\frac{\epsilon}{4}
\end{aligned}
$$

for these choices of $s$ and $t$, and in this sense, the integral over $X$ is well approximated by one over $K$.

A similar argument, using Lemma4.3 applied to $X_{0}$ and $K_{0}$, gives an $s_{3}$ such that for $s>s_{3}$

$$
\left|\int_{X_{0}}\left\langle\left(\phi_{1}\right)_{*} \tau, \frac{\tilde{\chi}_{s}^{*}\left(\sigma^{\tilde{m}}\right)}{\left\|\tilde{\chi}_{s}^{*}\left(\sigma^{\tilde{m}}\right)\right\|}\right\rangle d(v o l)-\int_{K_{0}}\left\langle\left(\phi_{1}\right)_{*} \tau, \frac{\tilde{\chi}_{s}^{*}\left(\sigma^{\tilde{m}}\right)}{\left\|\tilde{\chi}_{s}^{*}\left(\sigma^{\tilde{m}}\right)\right\|}\right\rangle d(v o l)\right|<\frac{\epsilon}{4}
$$

and so the integral over $X_{0}$ is well approximated by one over $K_{0}$.

Next, for any $0<t<1$ we can push forward by $\phi_{1-t}$ to rewrite the integral over $K$ as an integral over $K_{t}$ as follows. Recalling the definition of $\sigma_{s, t}^{m}$ from (3.14) we have

$$
\int_{K}\left\langle\tau, \frac{\sigma_{s, t(s)}^{m}}{\left\|\sigma_{s, t(s)}^{m}\right\|}\right\rangle d(v o l)=\int_{K_{t}}\left\langle\left(\phi_{1-t}\right)_{*} \tau, \frac{\tilde{\rho}_{s, t}^{*} \tilde{\chi}_{s}^{*}\left(\sigma^{\tilde{m}}\right)}{\left\|\tilde{\rho}_{s, t}^{*} \tilde{\chi}_{s}^{*}\left(\sigma^{\tilde{m}}\right)\right\|}\right\rangle d(v o l) .
$$

We then have the following:

$$
\begin{aligned}
\mid \int_{K_{t}}\left\langle\left(\phi_{1-t}\right)_{*} \tau, \frac{\tilde{\rho}_{s, t}^{*} \tilde{\chi}_{s}^{*}\left(\sigma^{\tilde{m}}\right)}{\left\|\tilde{\rho}_{s, t}^{*} \tilde{\chi}_{s}^{*}\left(\sigma^{\tilde{m}}\right)\right\|}\right\rangle d(\mathrm{vol}) & -\int_{K_{t}}\left\langle\left(\phi_{1-t}\right)_{*} \tau, \frac{\phi_{t}^{*} \tilde{\chi}_{s}^{*}\left(\sigma^{\tilde{m}}\right)}{\left\|\phi_{t}^{*} \tilde{\chi}_{s}^{*}\left(\sigma^{\tilde{m}}\right)\right\|}\right\rangle d(\mathrm{vol}) \mid \\
& \leq \int_{K_{t}}\left|\left\langle\left(\phi_{1-t}\right)_{*} \tau, \frac{\tilde{\rho}_{s, t}^{*} \tilde{\chi}_{s}^{*}\left(\sigma^{\tilde{m}}\right)}{\left\|\tilde{\rho}_{s, t}^{*} \tilde{\chi}_{s}^{*}\left(\sigma^{\tilde{m}}\right)\right\|}\right\rangle-\left\langle\left(\phi_{1-t}\right)_{*} \tau, \frac{\phi_{t}^{*} \tilde{\chi}_{s}^{*}\left(\sigma^{\tilde{m}}\right)}{\left\|\phi_{t}^{*} \tilde{\chi}_{s}^{*}\left(\sigma^{\tilde{m}}\right)\right\|}\right\rangle\right| d(\mathrm{vol}) \\
& =\int_{K_{t}}\left|\left\langle\left(\phi_{1-t}\right)_{*} \tau, \frac{\tilde{\rho}_{s, t}^{*} \tilde{\chi}_{s}^{*}\left(\sigma^{\tilde{m}}\right)}{\left\|\tilde{\rho}_{s, t}^{*} \tilde{\chi}_{s}^{*}\left(\sigma^{\tilde{m}}\right)\right\|}-\frac{\phi_{t}^{*} \tilde{\chi}_{s}^{*}\left(\sigma^{\tilde{m}}\right)}{\left\|\phi_{t}^{*} \tilde{\chi}_{s}^{*}\left(\sigma^{\tilde{m}}\right)\right\|}\right\rangle\right| d(v o l) \\
& \leq \int_{K_{t}}\left|\left(\phi_{1-t}\right)_{*} \tau\right|\left|\frac{\tilde{\rho}_{s, t}^{*} \tilde{\chi}_{s}^{*}\left(\sigma^{\tilde{m}}\right)}{\left\|\tilde{\rho}_{s, t}^{*} \tilde{\chi}_{s}^{*}\left(\sigma^{\tilde{m}}\right)\right\|}-\frac{\phi_{t}^{*} \tilde{\chi}_{s}^{*}\left(\sigma^{\tilde{m}}\right)}{\left\|\phi_{t}^{*} \tilde{\chi}_{s}^{*}\left(\sigma^{\tilde{m}}\right)\right\|}\right| d(v o l) \\
& \leq C \int_{K_{t}}\left|\frac{\tilde{\rho}_{s, t}^{*} \tilde{\chi}_{s}^{*}\left(\sigma^{\tilde{m}}\right)}{\left\|\tilde{\rho}_{s, t}^{*} \tilde{\chi}_{s}^{*}\left(\sigma^{\tilde{m}}\right)\right\|}-\frac{\phi_{t}^{*} \tilde{\chi}_{s}^{*}\left(\sigma^{\tilde{m}}\right)}{\left\|\phi_{t}^{*} \tilde{\chi}_{s}^{*}\left(\sigma^{\tilde{m}}\right)\right\|}\right| d(v o l)
\end{aligned}
$$

where the last inequality again uses the upper bound $\left|\left(\phi_{1-t}\right)_{*} \tau\right|<C$. Now let $\varepsilon=\frac{\epsilon}{2 C \operatorname{vol}\left(X_{t}\right)}$. (Note that volumes are equal for all fibers, i.e. $\operatorname{vol}(X)=\operatorname{vol}\left(X_{t}\right)$ for all $t$.) Applying Lemma 4.8 to this value of $\varepsilon$, we obtain a continuous monotone (non-increasing) function $t^{\prime \prime}(s)$ of $s$ such that for all $0<t<t^{\prime \prime}(s)$ and all $x \in K_{t}$ we have

$$
\left|\frac{\tilde{\rho}_{s, t}^{*} \tilde{\chi}_{s}^{*}\left(\sigma^{\tilde{m}}\right)}{\left\|\tilde{\rho}_{s, t}^{*} \tilde{\chi}_{s}^{*}\left(\sigma^{\tilde{m}}\right)\right\|}-\frac{\phi_{t}^{*} \tilde{\chi}_{s}^{*}\left(\sigma^{\tilde{m}}\right)}{\left\|\phi_{t}^{*} \tilde{\chi}_{s}^{*}\left(\sigma^{\tilde{m}}\right)\right\|}\right|<\frac{\epsilon}{4 C \operatorname{vol}\left(X_{t}\right)}
$$

which implies that the integral in (4.21) is less than $\epsilon / 4$.

Finally, let $t(s)=\min \left\{t^{\prime}(s), t^{\prime \prime}(s)\right\}$ be the minimum of the two continuous functions $t^{\prime}(s)$ and $t^{\prime \prime}(s)$ defined earlier. Then $t(s)$ is a continuous, positive, decreasing function of $s$, and the estimates in (4.19) and (4.21) hold for all $0<t<t(s)$. Let $s_{0}=\max \left\{s_{1}, s_{2}, s_{3}\right\}$. The triangle inequality then implies that the LHS of (4.16) is less than or equal to the sum of the left-hand sides of (4.18), (4.19), (4.20), and (4.21), each of which is less than $\epsilon / 4$ for $s>s_{0}$. This implies that, for this function $t(s)$ and this choice of $s_{0}$, the inequality (4.16) holds for $s>s_{0}$, and we are finished with the proof of Proposition 4.5 
In Section 3.2 we constructed for each $s$ and $t$ a complex structure $J_{s, t}$ on $X$ that is compatible with the symplectic structure. Recall also that the parameter $s \in[0, \infty)$ corresponds to the deformation of complex structures while the $t \in[0,1]$ parameter corresponds to the gradient-Hamiltonian flow from $X_{1}$ to $X_{0}$. Our final task is to construct the family $J_{s}$ of complex structures and the basis of sections $\sigma_{s}^{m}$ in the statement of our main Theorem 2.3. The basic idea behind the definition below is to let $s$ go to $\infty$ and $t$ go to 0 simultaneously in such a way that the convergence which is claimed in Theorem 2.3 occurs. Indeed, in Proposition 4.5 we constructed a continuous function $t(s)$ of $s$ which gives rise to certain key estimates for any $s>0$ and $0 \leq t<t(s)$. Thus, setting $J_{s}:=J_{s, t(s)}$ would (nearly) do the job; however, although we need $J_{0}$ to be the original complex structure (which is $J_{0,1}$ ) on $X$, our construction of $t(s)$ does not guarantee that $t(0)=1$. The solution to this problem is, roughly speaking, to first move along the gradient-Hamiltonian flow to $t_{0}$ while keeping $s=0$ before "turning on" the other deformation. More precisely, we make the following definition.

Definition 4.9. Let $t(s)$ denote the continuous function constructed in Proposition 4.5. For $s \in[0, \infty)$, we define

$$
J_{s}:= \begin{cases}J_{0,1+\left(t_{0}-1\right) s} & \text { if } 0 \leq s \leq 1 \text { and } \\ J_{s-1, t(s-1)} & \text { if } s>1 .\end{cases}
$$

Note that by construction $J_{0}=J_{0,1}$ is the original complex structure on $X$, and as $s \rightarrow \infty, J_{s}$ has the same convergence properties as $J_{s, t(s)}$. Moreover, by construction, the family $J_{s}$ is continuous with respect to the parameter $s$. We are finally ready to prove our main Theorem 2.3 .

Proof of Theorem 2.3 Consider the family $\left\{J_{s}\right\}_{s \in[0, \infty)}$ given in Definition 4.9 As already noted above, $\left\{J_{s}\right\}$ is a continuous family and $J_{0}=J_{0,1}$ is the original complex structure by construction. By Lemma 3.4, the pair $\left(\omega=\omega_{1}, J_{s}\right)$ is a Kähler structure on $X$ for each $s \in[0, \infty)$. Now define

$$
\sigma_{s}^{m}:= \begin{cases}\sigma_{0,1+\left(t_{0}-1\right) s}^{m} & \text { if } 0 \leq s \leq 1 \\ \sigma_{s-1, t(s-1)}^{m} & \text { if } s>1 .\end{cases}
$$

By construction, $\sigma_{s}^{m}$ is an element of $H^{0}\left(X, L, \bar{\partial}_{s}\right)$. Moreover, by definition $J_{s}$ and $\sigma_{s}^{m}$ have the same limiting properties as $J_{s, t(s)}$ and $\sigma_{s, t(s)}^{m}$, and for any interior point $m \in W_{0}$ it was shown in Proposition 4.5 that $\sigma_{s, t(s)}^{m}$ has the required limiting property of (2.2) in the statement of Theorem 2.3. Thus it remains only to ensure that for each fixed $s \mathrm{w}$ have a basis of $H^{0}\left(X, L, \bar{\partial}_{s}\right)$. By the limiting properties of the $\sigma_{s}^{m}$, we know that as $s$ goes to $\infty$, the supports of the sections $\sigma_{s}^{m}$ are increasingly concentrated in pairwise disjoint neighborhoods. It follows that the set $\left\{\sigma_{s}^{m}\right\}$ must be linearly independent for $s>s_{0}$ with $s_{0}$ sufficiently large. Since the complex manifolds $\left(X, J_{s}\right)$ and holomorphic line bundles $\left(L, \bar{\partial}_{s}\right)$ are isomorphic for all $s$, we also know that $\operatorname{dim} H^{0}\left(X, L, \bar{\partial}_{s}\right)$ is constant for all $s$, and in particular by assumption (h) in the statement of Theorem 2.3, we have $\operatorname{dim} H^{0}\left(X, L, \bar{\partial}_{s}\right)=\left|W_{0}\right|$ for all $s$. Thus for $s \geq s_{0}$ the set $\left\{\sigma_{s}^{m}\right\}$ is linearly independent and also spans, so it is a basis of $H^{0}\left(X, L, \bar{\partial}_{s}\right)$ as desired. For $0 \leq s \leq s_{0}$, following [7, Section 7.2] we extend the basis $\left\{\sigma_{s}^{m}\right\}_{m \in W_{0}}, s \geq s_{0}$, to bases of $H^{0}\left(X, L, \bar{\partial}_{s}\right)$ for $s$ satisfying $0 \leq s \leq s_{0}$ in a way that preserves the continuity in the parameter $s$. This family then satisfies all the required properties.

\section{TORIC DEGENERATIONS COMING FROM VALUATIONS AND NEWTON-OKOUNKOV BODIES}

In this section we will show that the toric degenerations coming from Newton-Okounkov bodies as in [8] can be used to create many examples of algebraic varieties $X$ with prequantum data $(\omega, J, L, h, \nabla)$ which satisfy the hypotheses of Theorem [2.3. This is the content of the main result of this section, Theorem 5.5.

We first very briefly recall the ingredients in the definition of a Newton-Okounkov body. For details we refer the reader to [11,15] and also [8]. We begin with the definition of a valuation (in our setting). We equip $\mathbb{Z}^{n}$ with a group ordering e.g. a lexicographic order. 
Definition 5.1. (1) Let $A$ be a $\mathbb{C}$ - algebra. A valuation on $A$ is a function

$$
\nu: A \backslash\{0\} \rightarrow \mathbb{Z}^{n}
$$

satisfying the following:

(a) $\nu(c f)=\nu(f)$ for all $f \in A \backslash\{0\}$ and $c \in \mathbb{C} \backslash\{0\}$,

(b) $\nu(f+g) \geq \min \{\nu(f), \nu(g)\}$ for all $f, g \in A \backslash\{0\}$ with $f+g \neq 0$.

(c) $\nu(f g)=\nu(f)+\nu(g)$ for all $f, g \in A \backslash\{0\}$.

(2) The image $\nu(A \backslash\{0\}) \subset \mathbb{Z}^{n}$ of a valuation $\nu$ on a $\mathbb{C}$-algebra $A$ is clearly a semigroup and is called the value semigroup of the pair $(A, \nu)$.

(3) Moreover, if in addition the valuation has the property that for any pair $f, g \in A \backslash\{0\}$ with same value $\nu(f)=\nu(g)$ there exists a non-zero constant $c \neq 0 \in \mathbb{C}$ such that either $\nu(g-c f)>\nu(g)$ or else $g-c f=0$ then we say that the valuation has one-dimensional leaves.

If $\nu$ is a valuation with one-dimensional leaves, then the image of $\nu$ is a sublattice of $\mathbb{Z}^{n}$ of full rank. Hence, by replacing $\mathbb{Z}^{n}$ with this sublattice if necessary, we will always assume without loss of generality that $\nu$ is surjective.

Given a variety $X$, there exist many possible valuations with one-dimensional leaves on its field of rational functions $\mathbb{C}(X)$. Strictly speaking, we do not need detailed knowledge of the construction in this paper, but we note for the reader's reference that the following example is the one which arises naturally in geometric contexts: for an $n$-dimensional variety $X$, a choice of an (ordered) coordinate system at a smooth point $p$ on $X$ gives a valuation on $\mathbb{C}(X)$ with one-dimensional leaves, essentially by computing the order of the zero or pole with respect to the coordinates. See e.g. [11, Examples 2.12 and 2.13 ] or [15] for details.

The following proposition is simple but fundamental [11, Proposition 2.6]:

Proposition 5.2. Let $\nu$ be a valuation on $\mathbb{C}(X)$ with one-dimensional leaves. Let $V \subset \mathbb{C}(X)$ be a finite-dimensional subspace of $\mathbb{C}(X)$. Then $\operatorname{dim}_{\mathbb{C}}(V)=|\nu(V \backslash\{0\})|$.

Let $X$ be a projective variety of dimension $n$ over $\mathbb{C}$ equipped with a very ample line bundle $L$. Let $E:=H^{0}(X, L)$ denote the space of global sections of $L$; it is a finite dimensional vector space over $\mathbb{C}$. The line bundle $L$ gives rise to the Kodaira map $\Phi_{E}$ of $E$, from $X$ to the projective space $\mathbb{P}\left(E^{*}\right)$. The assumption that $L$ is very ample implies that the Kodaira map $\Phi_{E}$ is an embedding.

Now let $E^{k}$ denote the image of the $k$-fold product $E \otimes \cdots \otimes E$ in $H^{0}\left(X, L^{\otimes k}\right)$ under the natural map given by taking the product of sections. (In general this map may not be surjective.) The homogeneous coordinate ring of $X$ with respect to the embedding $\Phi_{E}: X \hookrightarrow \mathbb{P}\left(E^{*}\right)$ can be identified with the graded algebra

$$
R=R(E)=\bigoplus_{k \geq 0} R_{k},
$$

where $R_{k}:=E^{k}$. This is a subalgebra of the ring of sections

$$
R(L)=\bigoplus_{k \geq 0} H^{0}\left(X, L^{\otimes k}\right) .
$$

For a fixed $\nu$ we now associate a semigroup $S(R) \subset \mathbb{N} \times \mathbb{Z}^{n}$ to $R$. First we identify $E=H^{0}(X, L)$ with a (finite-dimensional) subspace of $\mathbb{C}(X)$ by choosing a non-zero element $h \in E$ and mapping $f \in E$ to the rational function $f / h \in \mathbb{C}(X)$. Similarly, we can associate the rational function $f / h^{k}$ to an element $f \in R_{k}:=E^{k} \subseteq H^{0}\left(X, L^{\otimes k}\right)$. We define

$$
S=S(R)=S(R, \nu, h)=\bigcup_{k>0}\left\{\left(k, \nu\left(f / h^{k}\right)\right) \mid f \in E^{k} \backslash\{0\}\right\} .
$$

If $f \in R_{k}=E^{k}$ is a homogeneous element of degree $k$ we also define:

$$
\tilde{\nu}(f)=\left(k, \nu\left(f / h^{k}\right)\right) \text {. }
$$


Now define $C(R) \subseteq \mathbb{R} \times \mathbb{R}^{n}$ to be the cone generated by the semigroup $S(R)$, i.e., it is the smallest closed convex cone centered at the origin containing $S(R)$. We can now define the central object of interest.

Definition 5.3. Let $\Delta=\Delta(R)=\Delta(R, \nu)$ be the slice of the cone $C(R)$ at level 1, that is, $C(R) \cap\left(\{1\} \times \mathbb{R}^{n}\right)$, projected to $\mathbb{R}^{n}$ via the projection to the second factor $\mathbb{R} \times \mathbb{R}^{n} \rightarrow \mathbb{R}^{n}$. We have

$$
\Delta=\overline{\operatorname{conv}\left(\bigcup_{k>0}\left\{\frac{x}{k}:(k, x) \in S(R)\right\}\right)} .
$$

The convex body $\Delta$ is called the Newton-Okounkov body of $R$ with respect to the valuation $\nu$.

From now on, we place the additional assumption that:

$$
S \text { is finitely generated. }
$$

The above assumption is a rather strong condition on $(X, L, \nu)$ but it holds in many cases of importance. We note that it is possible to have a finitely generated semigroup $S$ for one choice of a valuation $\nu$ and a nonfinitely generated one for a different choice of $\nu$. From the above assumption it follows that the NewtonOkounkov body $\Delta(R)$ is a rational polytope. In this context, Anderson proved the following [2, Corollary 5.3].

Theorem 5.4. There is a flat family $\pi: X \rightarrow \mathbb{C}$ such that:

(a) For any $z \neq 0$ the fiber $X_{z}=\pi^{-1}(z)$ is isomorphic to $X$, and $\pi^{-1}\left(\mathbb{C}^{*}\right)$ is isomorphic to $X \times \mathbb{C}^{*}$. For the remainder of the discussion we fix an isomorphism $X \times \mathbb{C}^{*} \rightarrow \pi^{-1}\left(\mathbb{C}^{*}\right) \subset X$.

(b) The special fiber $X_{0}=\pi^{-1}(0)$ is isomorphic to $\operatorname{Proj}(\operatorname{gr} R) \cong \operatorname{Proj}(\mathbb{C}[S])$ and is equipped with an action of $\mathbb{T}=\left(\mathbb{C}^{*}\right)^{n}$, where $n=\operatorname{dim}_{\mathbb{C}} X$. The normalization of the variety $\operatorname{Proj}(\operatorname{gr} R)$ is the toric variety associated to the rational polytope $\Delta(R)$.

The explicit construction of the family $X$ in [2] depends on a choice of a so-called Khovanskii basis $\mathcal{B}=\left\{f_{i j}\right\}$ (cf. [8. Definition 8.1], and also see [12] for a general theory of Khovanskii bases). The set $\mathcal{B}$ also allows us to concretely embed $\mathcal{X}$ as a subvariety of $\mathcal{P} \times \mathbb{C}$ for an appropriate "large" projective space $\mathcal{P}$. Some of the details are relevant for our later discussions so we briefly recall the construction here; for details we refer to [8, Sections 8 and 9].

By assumption the semigroup $S \subset \mathbb{N} \times \mathbb{Z}^{n}$ is finitely generated. So we can find a finite set consisting of homogeneous elements in $R$ such that their valuations are a set of generators for $S$. More precisely, let $r>0$ be a positive integer and let $\mathcal{B}=\left\{f_{i j}\right\}$, for $1 \leq i \leq r, 1 \leq j \leq n_{i}=\operatorname{dim}\left(R_{i}\right)$, be a finite set of elements in $R$ satisfying the following properties:

(a) the $f_{i j}$ are homogeneous, with $f_{i j} \in R_{i}$ for all $1 \leq i \leq r, 1 \leq j \leq n_{i}$, and

(b) for each $i$, the collection $\left\{f_{i 1}, f_{i 2}, \ldots, f_{i n_{i}}\right\}$ is a vector space basis for $R_{i}$,

(c) the set of images $\tilde{\nu}(\mathcal{B})=\{\tilde{\nu}(f) \mid f \in \mathcal{B}\}$ generate $S=S(R)$,

For the remainder of this discussion we fix this "Khovanskii basis" $\mathcal{B}$.

We now describe more explicitly, in terms of the Khovanskii basis, the toric degeneration $X$ constructed in [2] and a concrete embedding of $\mathcal{X}$ into $\mathcal{P} \times \mathbb{C}$ for a suitable large projective space $\mathbb{C}$. Let $S_{d}:=S \cap\left(\{d\} \times \mathbb{Z}^{n}\right)$ denote the level- $d$ piece of the semigroup $S$. By Proposition 5.2, $\operatorname{dim}\left(E^{d}\right)=\left|\nu\left(E^{d}\right)\right|=\left|S_{d}\right|$, and since the $\left\{f_{i j}\right\}$ form a Khovanskii basis, for each $s \in S_{d}$ we know there exists some monomial $f_{11}^{\alpha_{11}} f_{12}^{\alpha_{12}} \cdots f_{r n_{r}}^{\alpha_{r n_{r}}}$ in the $\left\{f_{i j}\right\}^{\prime}$ 's, where $\sum_{i=1}^{r} i \sum_{j=1}^{n_{i}} \alpha_{i j}=d$, such that $\nu\left(f_{11}^{\alpha_{11}} f_{12}^{\alpha_{12}} \cdots f_{r n_{r}}^{\alpha_{r_{r}}}\right)=s$. So for each $s \in S$ we fix a choice of such exponents $\alpha_{s}:=\left(\alpha_{(i j), s}\right)$ such that the above holds. Then the set

$$
\left\{f_{11}^{\alpha_{(11), s}} f_{12}^{\alpha_{(12), s}} \cdots f_{r n_{r}}^{\alpha_{\left(r n_{r}\right), s}} \mid s \in S_{d}\right\}
$$

forms a basis for $E^{d}$. In [2], a collection of integers $w_{i j}$ are associated to the $f_{i j}$ in a certain way (for details see [2] and [8, Section 8]). Using these integers $w_{i j}$ and the above choices we can describe explicitly the toric degeneration $X$ and its embedding as follows. We first define a morphism $X \times \mathbb{C}^{*} \rightarrow \mathbb{P}\left(\left(E^{d}\right)^{*}\right) \times \mathbb{C}^{*}$ by 
expressing the Kodaira embedding $X \rightarrow \mathbb{P}\left(\left(E^{d}\right)^{*}\right)$ explicitly using the above basis for $E^{d}$. In coordinates we have

$$
(x, t) \mapsto\left(\left(t^{\sum_{i j} w_{i j} \alpha_{i j}} f_{11}(x)^{\alpha_{11}} \cdots f_{r n_{r}}(x)^{\alpha_{r n_{r}}} \mid \alpha_{i j}=\alpha_{(i j), s}, s \in S_{d}\right), t\right)
$$

Then the toric degeneration $X \subseteq \mathcal{P} \times \mathbb{C}$ is defined to be the closure of the image of (5.3). By its construction, $X$ is isomorphic to the fiber $X_{1}$ and $X_{0}$ is a toric variety [2, Corollary 5.3].

Note that the pullback to $X$ of the line bundle $L_{\mathcal{P}}$ over $\mathcal{P}$ is $L^{\otimes d}$ by construction. Given any prequantum data $\left(\omega_{\mathcal{P}}, L_{\mathcal{P}}, h_{\mathcal{P}}, \nabla_{\mathcal{P}}\right)$ on $\mathcal{P}=\mathbb{P}\left(\left(E^{d}\right)^{*}\right)$, it is clear that this data can be pulled back via the embedding (5.3) to prequantum data $\left(\omega, L^{\otimes d}, h, \nabla\right)$ on the line bundle $L^{\otimes d}$ over $X$.

We have the following, which is the main result of this section.

Theorem 5.5. Let $X$ be a smooth, irreducible complex algebraic variety with $\operatorname{dim}_{\mathbb{C}}(X)=n$, let $L$ be a very ample line bundle on $X$, and $E:=H^{0}(X, L)$. Then there exists a sufficiently large positive integer $d$ and prequantum data $\left(\omega_{\mathcal{P}}, h_{\mathcal{P}}, \nabla_{\mathcal{P}}\right)$ on $L_{\mathcal{P}} \rightarrow \mathcal{P}$ such that the family $X \subseteq \mathcal{P} \times \mathbb{C}$ constructed above is a toric degeneration of $X$ in the sense of Section 2 , and moreover, with respect to the pullback prequantum data $(\omega, h, \nabla)$ on $L^{\otimes d} \rightarrow X$, this toric degeneration satisfies all the required hypotheses $(a)-(h)$, and thus gives 'convergence of polarization' in these cases.

Proof of Theorem 5.5 The fact that $X \subseteq \mathcal{P} \times \mathbb{C}$ is a toric degeneration satisfying the hypotheses (a) and (b) of Theorem 2.3 follows from the construction in [2] and is shown in [8]. Moreover, in [8, Section 9] an appropriate Kähler structure $\omega_{\mathcal{P}}$ on $\mathcal{P}$ is constructed which satisfies condition (d). Indeed, the construction of $\omega_{\mathcal{P}}$ is by pulling back a Fubini-Study form associated to a hermitian structure on an (even larger) projective space, and in particular - by pulling back the standard prequantum data on a projectivization of a vector space equipped with a hermitian structure - it is clear that we can construct the prequantum data $\left(\omega_{\mathcal{P}}, h_{\mathcal{P}}, \nabla_{\mathcal{P}}\right)$ compatible with $\omega_{\mathcal{P}}$. Now the hypothesis (c) follows by construction, since the relevant prequantum data are defined via pullbacks.

We also claim that hypothesis (e) holds in our situation. Indeed, by our choice of basis (5.2) of $E^{d}$, it follows that our embedding (5.3) has the property that the coordinates of the embedding correspond exactly to elements of $S_{d}$. In particular, it follows from [2, Proposition 5.1] and [8, Section 8] that the special fiber $X_{0}$ is exactly the closure of a $\mathbb{T}_{0}$-orbit through a point of the form $[1: 1: \cdots: 1]$, where $\mathbb{T}_{0} \cong\left(\mathbb{C}^{*}\right)^{n}$ acts by weight $s \in S_{d} \subseteq \mathbb{Z}^{n}$ on the coordinate associated to $s \in S_{d}$. It also follows that the set $W_{0}$ defined in (2.1) is precisely $S_{d} \subseteq \mathbb{Z}^{n}$, and thus hypothesis (h) follows from the fact, already observed above, that $\operatorname{dim} E^{d}=\operatorname{dim} H^{0}\left(X, L^{\otimes d}\right)=\left|S_{d}\right|$.

Next, it is well-known [9. Chapter II, Section 5, Exercise 5.14(b)] that for sufficiently large $d \gg 0$, we have $E^{d}=H^{0}\left(X, L^{\otimes d}\right)$, so in particular $\mathbb{P}\left(\left(E^{d}\right)^{*}\right)=\mathbb{P}\left(\left(H^{0}\left(X, L^{\otimes d}\right)\right)^{*}\right)$ and the restriction map $H^{0}\left(\mathcal{P}, L_{\mathcal{P}}\right)=$ $H^{0}\left(\mathbb{P}\left(H^{0}\left(X, L^{\otimes d}\right)^{*}\right), \mathcal{O}(1)\right) \rightarrow H^{0}\left(X, L^{\otimes d}\right)$ is surjective, so $X$ satisfies hypothesis (f) for sufficiently large $d$.

Finally, we claim that for sufficiently large $d \gg 0$ we also have that hypothesis (g) holds. By definition of the torus $\mathbb{T}_{0}$, the graded semigroup $S$ generates $\mathbb{Z} \times M$ where $M$ is the character lattice of $\mathbb{T}_{0}$ (and can be identified with $\left.\left(\mathfrak{t}_{0}^{*}\right)_{\mathbb{Z}}\right)$. It is easy to see that in this situation there exists $d$ sufficiently large such that $S_{d}=\nu\left(H^{0}\left(X, L^{\otimes d}\right) \backslash\{0\}\right)$ generates $M$. Since $S_{d}$ is contained in $\iota^{*}\left(\left(\mathfrak{t}_{\mathcal{P}}^{*}\right)_{\mathbb{Z}}\right)$ by what we said above, the hypothesis (g) follows for $d$ sufficiently large.

Thus, by taking $d$ large enough so that both of the last two phenomena occur, we obtain the results claimed in the theorem.

Finally, in the situation of an integrable system coming from a toric degeneration arising from a NewtonOkounkov body, the sections $\sigma_{s, t}^{m}$ we construct in $\$ 3.4$ form a basis of $H^{0}\left(X, L, \bar{\partial}_{s, t}\right)$ for all values of $s$ and $t$. In particular, in this case we do not need to "extend the basis" non-constructively as in the last sentence of the proof of Theorem 2.3 , at the very end of $\$ 4$ 
Theorem 5.6. Let the notation be as in Theorem 5.5 Then for any fixed $s \geq 0, t \neq 0$, the set $\left\{\sigma_{s, t}^{m} \mid m \in W_{0}\right\}$ constructed in Definition 3.14 is linearly independent. In particular, the set $\left\{\sigma_{s}^{m} \mid m \in W_{o}\right\}$ constructed in (4.23) is a basis for $H^{0}\left(X, L^{d}, \bar{\partial}^{s}\right)$ for every value of $s \geq 0$.

Proof. By Definition 3.14, and since $\tilde{\phi}_{1-t}$ is an isomorphism of line bundles (see Lemma 3.7), it suffices to show that the $\tilde{\rho}_{s, t}^{*} \tilde{\chi}_{s}^{*} \sigma^{\tilde{m}}$ are linearly independent in $H^{0}\left(X_{t}, L_{t}\right)$. By [7, Proposition 6.1(2)], for each $s \geq 0$ and each $0<t \leq 1$ we have a diffeomorphism $\underline{\chi}_{s, t}: X_{t} \rightarrow X_{t}$ such that the following diagram commutes:

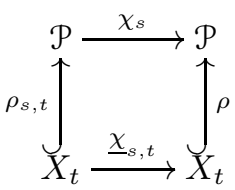

where $\rho$ is the standard embedding of $X_{t}$ into $\mathcal{P}$ as a complex manifold, and such that $\left(X_{t}, \rho_{s, t}^{*} \omega_{\mathcal{P}}, \chi_{s}^{*} J_{\mathcal{P}}\right)$ is Kähler. (Note that with our identifications the map $\chi_{0}$ in [7] is the identity.) The $\rho_{s, t}$ appearing in the diagram above are the embeddings of Proposition 3.2. Similarly, by [7. Proposition 6.3(2)] the map $\underline{\chi}_{s, t}$ lifts to a map $\underline{\tilde{\chi}}_{s, t}: L_{t} \rightarrow L_{t}$ such that

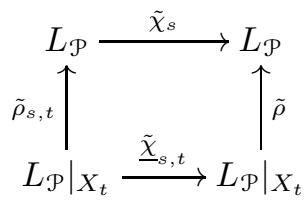

commutes, where $\tilde{\rho}_{s, t}$ are the maps in Lemma 3.8. Then $\left.\tilde{\chi}_{s}^{*} \sigma^{\tilde{m}}\right|_{X_{t}}=\underline{\tilde{\chi}}_{s, t}^{*}\left(\left.\sigma^{\tilde{m}}\right|_{X_{t}}\right)$. Since the $\tilde{\chi}_{s, t}$ are line bundle isomorphisms, it will suffice to show that the $\left.\sigma^{\tilde{m}}\right|_{X_{t}}$ 's are linearly independent.

To see this, recall the construction of the embedding of $X \times \mathbb{C}^{*}$ into $\mathcal{P} \times \mathbb{C}$. The sections

$$
\left\{f_{s}=f_{11}^{\alpha_{(11), s}} f_{12}^{\alpha_{(12), s}} \cdots f_{r n_{r}}^{\alpha_{\left(n_{r}\right), s}} \mid s \in S_{d}\right\}
$$

from (5.2) form a basis for the global sections of $\mathcal{O}(1)=L_{\mathcal{P}}$ on the projective space $\mathcal{P}=\mathbb{P}\left(\left(E^{d}\right)^{*}\right)$. The embedding of the family $X \times \mathbb{C}^{*} \subset \mathcal{X}$ in $\mathcal{P} \times \mathbb{C}$ in this basis is given by the map in (5.3) whose components are $t^{\sum_{i j} w_{i j} \alpha_{i j}} f_{s}, s \in S_{d}$. Since the line bundle is $\mathcal{O}(1)$, the functions $\sigma^{\tilde{m}}$ in the basis of its holomorphic sections are simply the coordinate functions on $\mathcal{P}$, and so the section $\left.\sigma^{\tilde{m}}\right|_{X_{t}}$ corresponds to one of the coordinates $t^{\sum_{i j} w_{i j} \alpha_{i j}} f_{s}$.

By construction, for fixed $t \neq 0$ the values of the valuation $\tilde{v}$ on the components $t^{\sum_{i j} w_{i j} \alpha_{i j}} f_{s}$ are distinct. Since elements with distinct values of valuation are linearly independent, it follows that, for any fixed $t \neq 0$, the sections $\left\{\left.\sigma^{\tilde{m}}\right|_{X_{t}} \mid m \in W_{0}\right\}$ are linearly independent, as desired.

\section{REFERENCES}

[1] M. Abreu. Kähler geometry of toric varieties and extremal metrics. Internat. J. Math., 9(6):641-651, 1998.

[2] D. Anderson. Okounkov bodies and toric degenerations. Math. Ann., 356(3):1183-1202, 2013.

[3] T. Baier, C. Florentino, J. M. Mourão, and J. P. Nunes. Toric Kähler metrics seen from infinity, quantization and compact tropical amoebas. J. Differential Geom., 89(3):411-454, 2011.

[4] V. Guillemin. Kaehler structures on toric varieties. J. Differential Geom., 40(2):285-309, 1994.

[5] V. Guillemin. Moment maps and combinatorial invariants of Hamiltonian $T^{n}$-spaces, volume 122 of Progress in Mathematics. Birkhäuser Boston, Inc., Boston, MA, 1994.

[6] M. D. Hamilton. The quantization of a toric manifold is given by the integer lattice points in the moment polytope. In Toric topology, volume 460 of Contemp. Math., pages 131-140. Amer. Math. Soc., Providence, RI, 2008.

[7] M. D. Hamilton and H. Konno. Convergence of Kähler to real polarizations on flag manifolds via toric degenerations. J. Symplectic Geom., 12(3):473-509, 2014.

[8] M. Harada and K. Kaveh. Integrable systems, toric degenerations and Okounkov bodies. Invent. Math., 202(3):927-985, 2015.

[9] R. Hartshorne. Algebraic geometry. Springer-Verlag, New York-Heidelberg, 1977. Graduate Texts in Mathematics, No. 52. 
[10] K. Kaveh. Crystal bases and Newton-Okounkov bodies. Duke Math. J., 164(13):2461-2506, 2015.

[11] K. Kaveh and A. G. Khovanskii. Newton-Okounkov bodies, semigroups of integral points, graded algebras and intersection theory. Ann. of Math. (2), 176(2):925-978, 2012.

[12] K. Kaveh and C. Manon Khvanskii bases, higher rank valuations and tropical geometry. arXiv:1610.00298

[13] V. A. Kiritchenko, E. Yu. Smirnov and V. A. Timorin Schubert calculus and Gelfand-Tsetlin polytopes. Uspekhi Mat. Nauk 67 (2012), no. 4(406), 89-128; translation in Russian Math. Surveys 67 (2012), no. 4, 685-719.

[14] M. Kogan and E. Miller Toric degeneration of Schubert varieties and Gelfand-Tsetlin polytopes. Adv. Math. 193 (2005), no. 1, 1-17.

[15] R. Lazarsfeld and M. Mustață. Convex bodies associated to linear series. Ann. Sci. Éc. Norm. Supér. (4), 42(5):783-835, 2009.

[16] T. Nishinou, Y. Nohara, and K. Ueda. Toric degenerations of Gelfand-Cetlin systems and potential functions. Adv. Math., 224(2):648-706, 2010

[17] J. P. Nunes. Degenerating kähler structures and geometric quantization. Rev. Math. Phys., 26, 2014.

[18] A. Okounkov Brunn-Minkowski inequality for multiplicities. Invent. Math. 125 (1996), no. 3, 405-411.

[19] A. Okounkov Why would multiplicities be log-concave? The orbit method in geometry and physics (Marseille, 2000), 329-347, Progr. Math., 213, Birkhäuser Boston, Boston, MA, 2003.

[20] W.-D. Ruan. Lagrangian torus fibration of quintic hypersurfaces. I. Fermat quintic case. In Winter School on Mirror Symmetry, Vector Bundles and Lagrangian Submanifolds (Cambridge, MA, 1999), volume 23 of AMS/IP Stud. Adv. Math., pages 297-332. Amer. Math. Soc., Providence, RI, 2001.

[21] T. Tao. Introduction to Measure Theory, volume 126 of Graduate Studies in Mathematics. American Mathematical Society, 2011.

[22] N. M. J. Woodhouse. Geometric quantization. Oxford Mathematical Monographs. The Clarendon Press, Oxford University Press, New York, second edition, 1992. Oxford Science Publications.

Department of Math and Computer Science, Mount Allison University, 67 York St., Sackville, NB, E4L 1E6, CANADA

E-mail address: mhamiltonemta.ca

Department of Mathematics and Statistics, McMaster University, 1280 Main Street West, Hamilton, Ontario L8S4K1, CANADA

E-mail address: Megumi.Harada@math.mcmaster.ca

URL:http://www.math.mcmaster.ca/Megumi.Harada/

Department of Mathematics, University of Pittsburgh, 301 Thackeray Hall, Pittsburgh, PA, 15260, USA

E-mail address: kavehepitt.edu

URL:http://www.pitt.edu/ kaveh/ 